Abstracted/indexed in Academic Search Complete, Asia Journals Online, Bangladesh Journals Online, Biological Abstracts, BIOSIS Previews, CAB

Abstracts, Current Abstracts, Directory of Open Access Journals, EMBASE/Excerpta Medica, Google Scholar, HINARI (WHO), International

Pharmaceutical Abstracts, Open J-gate, Science Citation Index Expanded, SCOPUS and Social Sciences Citation Index;

ISSN: $1991-0088$

\title{
Chemical and bioactive diversities of marine sponge Neopetrosia
}

\section{Haitham Qaralleh}

Department of Medical Support, Al-Balqa Applied University, Al-Karak University College, Al-Karak, Jordan.

\begin{tabular}{|c|c|}
\hline \multicolumn{2}{|l|}{ Article Info } \\
\hline Received: & 26 January 2016 \\
\hline Accepted: & 21 March 2016 \\
\hline Available Online: & 3 April 2016 \\
\hline \multicolumn{2}{|c|}{ DOI: 10.3329/bjp.v11i2.26611 } \\
\hline \multicolumn{2}{|l|}{ Cite this article: } \\
\hline \multicolumn{2}{|c|}{$\begin{array}{l}\text { Qaralleh H. Chemical and bioactive } \\
\text { diversities of the marine sponge Neo- } \\
\text { petrosia. Bangladesh J Pharmacol. } \\
\text { 2016; 11: 433-52. }\end{array}$} \\
\hline
\end{tabular}

\begin{abstract}
The marine sponge Neopetrosia contains about 27 species that is highly distributed in Indian Ocean, Atlantic Ocean (Caribbean Sea) and Pacific Ocean. It has proven to be valuable to the discovery of medicinal products due to the presence of various types of compounds with variable bioactivities. More than 85 compounds including alkaloids, quinones, sterols and terpenoids were isolated from this genus. Moreover, the crude extracts and the isolated compounds revealed activities such as antimicrobial, anti-fouling, anti-HIV, cytotoxic, anti-tumor, anti-oxidant, anti-protozoal, anti-inflammatory. Because only 9 out of 27 species of the genus Neopetrosia have been chemically studied thus far, there are significant opportunities to find out new chemical constituents from this genus.
\end{abstract}

\section{Introduction}

Marine sponges represent the major rich organisms with promising active pharmaceutical metabolites. The interest for drugs discovery in sponges has started since 1950s due to the discovery of the nucleosides spongothymidine and spongouridine from the marine sponge Cryptotethya crypta (Laport et al., 2009). Both metabolites were later developed to ara-C, the first marine-derived anti-cancer agent and the antiviral drug ara-A (Proksch et al., 2002). Later, several promising metabolites were discovered from marine sponges with different biological activities including antimicrobial and anti-cancer (Mayer et al., 2013). So far, more than $36 \%$ of the metabolites discovered from marine organisms were isolated from the sponges.

Neopetrosia is a genus of marine sponge that belongs to the phylum Porifera, the class Demospongia, of the order Haploscleridae, and family Petrosiidae. The genus was established by the Max Walker de Laubenfels in 1932. It contains about 27 species that is highly distributed in Indian Ocean, Atlantic Ocean (Caribbean Sea) and Pacific Ocean. Neopetrosia has received great attention in natural product chemistry. Several studies have been conducted lead to report the isolation of more than 85 metabolites. Therefore, the aim of this paper is to review the Neopetrosia genus, primarily focusing on their phytochemical characteristics and their biological activities.

Sponge species is identified based on the external morphological characteristics and spicules and skeleton characteristics. However, sponges are among the most difficult organisms to identify. Misidentification of this organism is common (Hooper et al., 2000; Qaralleh et al., 2011). Misidentification of sponges may lead to failure in the prediction of the chemical compo-sitions. Recently, many species belong to the Xestospongia or Petrosia genera were transferred to Neopetrosia genus.

In this study, World Register of Marine Species (www.marinespecies.org) was used to get the species scientific and synonymised names (Table I). Both names were used as a search keys in order to find the relevant literature about each species. The literature was collected by searching the major scientific databases including Marinlit, PubMed, SciFinder, Science direct, Scopus, Medline and Google Scholar. The data were then organised in Table which represented the species name, isolated compounds, place of collection and the bioactivities. 
Table I

Scientific names and synonym (s) for neopetrosia species

\begin{tabular}{|c|c|c|c|}
\hline & Species & Synonym (s) & Distribution \\
\hline 1 & Neopetrosia carbonaria (Lamarck, 1814) & $\begin{array}{l}\text { Adocia carbonaria (Lamarck, 1814) } \\
\text { Oceanapia carbonaria (Lamarck, 1814) } \\
\text { Pachychalina carbonaria (Lamarck, 1814) } \\
\text { Pellina carbonaria (Lamarck, 1814) } \\
\text { Spongia carbonaria Lamarck, } 1814 \\
\text { Thalysias carbonaria (Lamarck, 1814) } \\
\text { Xestospongia carbonaria (Lamarck, 1814) }\end{array}$ & Caribbean Sea \\
\hline 2 & Neopetrosia chaliniformis (Thiele, 1899) & $\begin{array}{l}\text { Petrosia (Petrosia) chaliniformis (Thiele, 1899) } \\
\text { Petrosia chaliniformis (Thiele, 1899) }\end{array}$ & Indonesia \\
\hline 3 & $\begin{array}{l}\text { Neopetrosia compacta (Ridley and } \\
\text { Dendy, 1886) }\end{array}$ & $\begin{array}{l}\text { Petrosia similis var. compacta (Ridley and } \\
\text { Dendy, 1886) }\end{array}$ & Philippines \\
\hline 4 & Neopetrosia contignata (Thiele, 1899) & $\begin{array}{l}\text { Haliclona contignata (Thiele, 1899) } \\
\text { Petrosia contignata (Thiele, 1899) }\end{array}$ & $\begin{array}{l}\text { Indonesia } \\
\text { East African Coral Coast } \\
\text { Gulf of Aden } \\
\text { Southern Red Sea }\end{array}$ \\
\hline 5 & Neopetrosia cylindrica (Lamarck, 1815) & $\begin{array}{l}\text { Alcyonium cylindricum (Lamarck, 1815) } \\
\text { Xestospongia cylindrica (Lamarck, 1815) }\end{array}$ & Caribbean Sea \\
\hline 6 & Neopetrosia delicatula (Dendy, 1905) & Petrosia similis var. delicatula (Dendy, 1905) & $\begin{array}{l}\text { Sri Lanka } \\
\text { South India }\end{array}$ \\
\hline 7 & Neopetrosia densissima (Wilson, 1904) & Petrosia similis var. densissima (Wilson, 1904) & Galapagos \\
\hline 8 & $\begin{array}{l}\text { Neopetrosia dominicana (Pulitzer-Finali, } \\
\text { 1986) }\end{array}$ & Xestospongia dominicana (Pulitzer-Finali, 1986) & $\begin{array}{l}\text { Dominican Republic } \\
\text { Greater Antilles }\end{array}$ \\
\hline 9 & Neopetrosia exigua (Kirkpatrick, 1900) & $\begin{array}{l}\text { Haliclona exigua (Kirkpatrick, 1900) } \\
\text { Neopetrosia pandora (de Laubenfels, 1954) } \\
\text { Petrosia exigua (Kirkpatrick, 1900) } \\
\text { Xestospongia exigua (Kirkpatrick, 1900) } \\
\text { Xestospongia pacifica (Kelly Borges and } \\
\text { Bergquist, 1988) }\end{array}$ & $\begin{array}{l}\text { Indian Ocean } \\
\text { Papua New Guinea } \\
\text { Singapore Strait } \\
\text { East African Coral Coast }\end{array}$ \\
\hline 10 & Neopetrosia granulosa (Wilson, 1925) & Petrosia similis var. granulosa (Wilson, 1925) & Philippines \\
\hline 11 & Neopetrosia halichondrioides Dendy, 1905 & $\begin{array}{l}\text { Petrosia similis var.halichondrioides (Dendy, } \\
\text { 1905) }\end{array}$ & $\begin{array}{l}\text { Sri Lanka } \\
\text { South India }\end{array}$ \\
\hline 12 & $\begin{array}{l}\text { Neopetrosia massa (Ridley \& Dendy, } \\
\text { 1886) }\end{array}$ & $\begin{array}{l}\text { Petrosia similis var. massa (Ridley and Dendy, } \\
\text { 1886) }\end{array}$ & $\begin{array}{l}\text { Falkland Islands } \\
\text { Malvinas/Falklands }\end{array}$ \\
\hline 13 & Neopetrosia perforata (Lévi, 1959) & Haliclona perforata (Lévi, 1959) & $\begin{array}{l}\text { Gulf of Guinea Islands } \\
\text { Sao Tome and Principe ex- } \\
\text { clusive economic zone }\end{array}$ \\
\hline 14 & $\begin{array}{l}\text { Neopetrosia problematica (de Laubenfels, } \\
\text { 1930) }\end{array}$ & $\begin{array}{l}\text { Dictyonella problematica (de Laubenfels, 1930) } \\
\text { Haliclona problematica (de Laubenfels, 1930) } \\
\text { Prianos problematicus (de Laubenfels, 1930) }\end{array}$ & Northern California \\
\hline 15 & $\begin{array}{l}\text { Neopetrosia proxima (Duchassaing and } \\
\text { Michelotti, 1864) }\end{array}$ & $\begin{array}{l}\text { Densa araminta (de Laubenfels, 1934) Thalysi- } \\
\text { as proxima (Duchassaing and Michelotti, } \\
\text { 1864) } \\
\text { Xestospongia proxima (Duchassaing and Mi- } \\
\text { chelotti, 1864) }\end{array}$ & $\begin{array}{l}\text { Caribbean Sea } \\
\text { North Atlantic Ocean }\end{array}$ \\
\hline 16 & Neopetrosia rava (Thiele, 1899) & Petrosia rava (Thiele, 1899) & Indonesia \\
\hline 17 & Neopetrosia retiderma (Dendy, 1922) & $\begin{array}{l}\text { Halichondria retiderma (Dendy, 1922) } \\
\text { Haliclona retiderma (Dendy, 1922) }\end{array}$ & $\begin{array}{l}\text { Seychelles } \\
\text { Indian Ocean }\end{array}$ \\
\hline 18 & $\begin{array}{l}\text { Neopetrosia rosariensis (Zea and } \\
\text { Rützler, 1983) }\end{array}$ & $\begin{array}{l}\text { Xestospongia rosariensis (Zea and Rützler, } \\
\text { 1983) }\end{array}$ & $\begin{array}{l}\text { Caribbean Sea } \\
\text { North Atlantic Ocean }\end{array}$ \\
\hline
\end{tabular}

According to World Register of Marine Species 


\section{Table I}

Scientific names and synonym (s) for neopetrosia species (Cont.)

\begin{tabular}{|c|c|c|c|}
\hline & Species & Synonym (s) & Distribution \\
\hline 19 & Neopetrosia sapra (de Laubenfels, 1954) & Xestospongia sapra (de Laubenfels, 1954) & $\begin{array}{l}\text { East Caroline Islands } \\
\text { Micronesia }\end{array}$ \\
\hline 20 & Neopetrosia seriata (Hentschel, 1912) & $\begin{array}{l}\text { Petrosia seriata (Hentschel, 1912) } \\
\text { Petrosia similis var. seriata (Hentschel, 1912) }\end{array}$ & $\begin{array}{l}\text { Arafura Sea } \\
\text { Indonesian exclusive Eco- } \\
\text { nomic Zone } \\
\text { Southern Vietnam } \\
\text { Vietnamese exclusive Eco- } \\
\text { nomic Zone }\end{array}$ \\
\hline 21 & $\begin{array}{l}\text { Neopetrosia similis (Ridley and Dendy, } \\
\text { 1886) }\end{array}$ & $\begin{array}{l}\text { Chalina similis (Ridley and Dendy, 1886) } \\
\text { Petrosia similis (Ridley and Dendy, 1886) }\end{array}$ & $\begin{array}{l}\text { Agulhas Bank } \\
\text { Eastern Philippines } \\
\text { Philippines exclusive eco- } \\
\text { nomic zone } \\
\text { South African exclusive } \\
\text { economic zone } \\
\text { South India, Sri Lanka }\end{array}$ \\
\hline 22 & $\begin{array}{l}\text { Neopetrosia subtriangularis } \\
\text { (Duchassaing, } \\
\text { 1850) }\end{array}$ & $\begin{array}{l}\text { Haliclona doria (de Laubenfels, 1936) } \\
\text { Haliclona longleyi (de Laubenfels, 1932) } \\
\text { Haliclona subtriangularis (Duchassaing and } \\
\text { Michelotti, 1864) } \\
\text { Neopetrosia longleyi (De Laubenfels, 1932) } \\
\text { Pachychalina rugosa (Duchassaing and Mi- } \\
\text { chelotti, 1864) } \\
\text { Pachychalina rugosa var. rubens (Arndt, 1927) } \\
\text { Schmidtia aulopora (Schmidt, 1870) } \\
\text { Spongia subtriangularis (Duchassaing, 1850) } \\
\text { Thalysias rugosa (Duchassaing and Michelotti, } \\
\text { 1864) } \\
\text { Thalysias subtriangularis (Duchassaing, 1850) } \\
\text { Thalysias subtriangularis var. cylindrica } \\
\text { (Duchassaing and Michelotti, 1864) } \\
\text { Thalysias subtriangularis var. lyriformis } \\
\text { (Duchassaing and Michelotti, 1864) } \\
\text { Xestospongia subtriangularis (Duchassaing, } \\
\text { 1850) }\end{array}$ & $\begin{array}{l}\text { Bahamas } \\
\text { Caribbean Sea } \\
\text { Caribbean Sea } \\
\text { Netherlands } \\
\text { Netherlands Antilles } \\
\text { United States }\end{array}$ \\
\hline 23 & Neopetrosia tenera (Carter, 1887) & Thalysias tenera (Carter, 1887) & $\begin{array}{l}\text { Andaman and Nicobar } \\
\text { Islands } \\
\text { Myanmar }\end{array}$ \\
\hline 24 & $\begin{array}{l}\text { Neopetrosia truncata (Ridley and } \\
\text { Dendy, 1886) }\end{array}$ & Petrosia truncata (Ridley and Dendy, 1886) & Philippines \\
\hline 25 & Neopetrosia tuberosa (Dendy, 1922) & $\begin{array}{l}\text { Haliclona tuberosa (Dendy, 1922) } \\
\text { Oceanapia tuberosa (Dendy, 1922) } \\
\text { Reniera tuberosa (Dendy, 1922) }\end{array}$ & $\begin{array}{l}\text { Indian Ocean } \\
\text { Saya de Malha } \\
\text { Seychelles } \\
\text { Cargados Carajos/Tromelin } \\
\text { Island } \\
\text { Western Arabian Sea }\end{array}$ \\
\hline 26 & $\begin{array}{l}\text { Neopetrosia vanilla (de Laubenfels, } \\
\text { 1930) }\end{array}$ & $\begin{array}{l}\text { Haliclona vanilla (de Laubenfels, 1930) } \\
\text { Xestospongia vanilla (de Laubenfels, 1930) }\end{array}$ & $\begin{array}{l}\text { California } \\
\text { North Pacific Ocean }\end{array}$ \\
\hline 27 & Neopetrosia zumi (Ristau, 1978) & $\begin{array}{l}\text { Haliclona (Reniera) zumi (Ristau, 1978) } \\
\text { Toxadocia zumi (Ristau, 1978) }\end{array}$ & $\begin{array}{l}\text { California } \\
\text { North Pacific Ocean }\end{array}$ \\
\hline
\end{tabular}




\section{Chemical Composition}

More than 85 compounds have been isolated from Neopetrosia species. These compounds were classified into alkaloids, quinones, sterols and terpenoids (Table II).

\section{Alkaloids}

More than 44 alkaloids have been isolated. These alkaloids were macrocyclic quinolizidines, 3-alkylpyridine alkaloids, pyridoacridine alkaloids and others.

\section{Macrocyclic quinolizidines}

Nineteen macrocyclic quinolizidines alkaloids have been reported from this genus. Several macrocyclic quinolizidines alkaloids (1-13 and 16-19) have been isolated from N. exigua and only two have been isolated from $N$. similes (14 and 15).

Four macrocyclic quinolizidines were isolated from Australian sponge $N$. exigua (Xestospongia exigua) namely xestospongin A (1), B (2), C (3) and D (4) (Nakagawa et al., 1984). Several araguspongines alkaloids were obtained from a red sea sponge $N$. exigua (Haliclona exigua) including (+)-araguspongine A (5), (+) -araguspongine B (6), (+)-araguspongine C (7), (+)araguspongine D (1), (-)-araguspongine E (8), and (+)xestospongin B (9) (Venkateswarlu et al., 1994; Venkateswara et al., 1998). (+)-araguspongine K (10) and (+)-araguspongine $\mathrm{L}(\mathbf{1 1})$ were isolated from a red sea sponge N. exigua (Xestospongia exigua) (Orabi et al., 2002). Araguspongin $C(12)$ was found from $n$-butanol

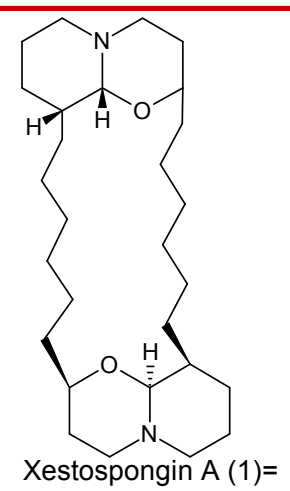

(+)-Araguaspongin D<smiles>O[C@@]12CCCCCC[C@H]3CCN4CCC[C@@H](CCCCCC[C@H]5CCN(CCC1)C2O5)O[C@H]4O3</smiles>

(+)-Araguspongine A (5)<smiles>C1CCC[C@@H]2CCCN3CCC[C@@H](CCCCCC[C@H]4CCCN5CCC(CC1)O[C@H]45)[C@H]3O2</smiles>

(+)-Xestospongin B (9)

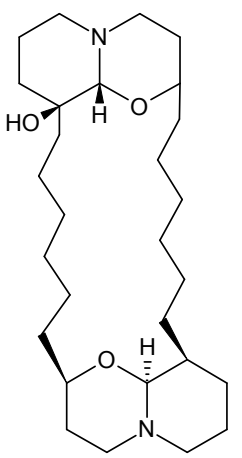

Xestospongin B (2)

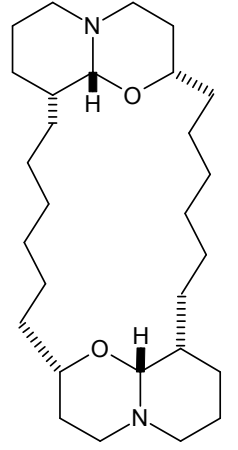

$(+)$-Araguspongine $B(6)$

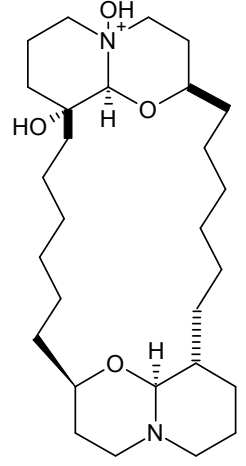

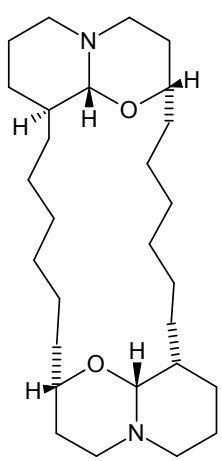

Xestospongin $\mathrm{C}(3)$

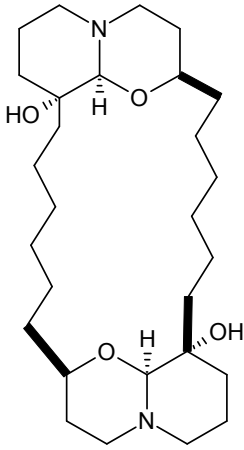

6) $(+$

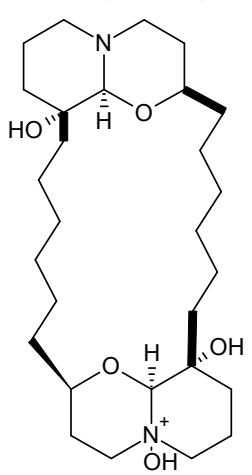

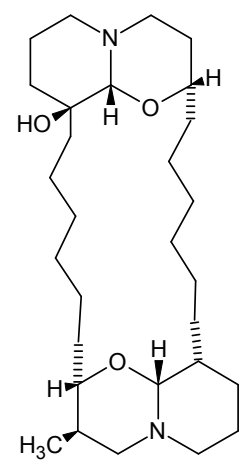

Xestospongin $\mathrm{D}(4)$

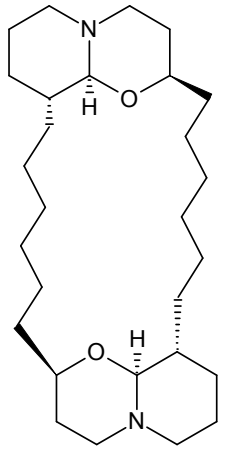

(-)-Araguspongine E (8)

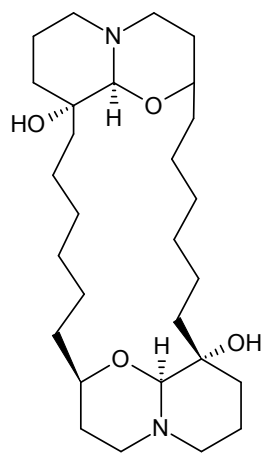

(+)-Araguspongine K (10) (+)-Araguspongine $L$ (11) Araguspongine $C$ (12) 


\section{Table II}

Chemical constituent of the Neopetrosia genus

\begin{tabular}{|c|c|c|c|}
\hline No. & Compound class and name & Source & Reference \\
\hline \multirow{2}{*}{\multicolumn{4}{|c|}{$\begin{array}{l}\text { Alkaloids } \\
\text { Macrocyclic quinolizidines }\end{array}$}} \\
\hline & & & \\
\hline 1 & $\begin{array}{l}\text { Xestospongin A } \\
((+) \text {-araguspongine D) }\end{array}$ & N. exigua (Xestospongia exigua) & $\begin{array}{l}\text { Nakagawa et al., 1984; Venkateswarlu et al., 1994, Ven- } \\
\text { kateswara et al., 1998; Liu et al., 2004; Li et al., } 2011\end{array}$ \\
\hline 2 & Xestospongin B & N. exigua (Xestospongia exigua) & Nakagawa et al., 1984 \\
\hline 3 & Xestospongin $\mathrm{C}$ & N. exigua (Xestospongia exigua) & Nakagawa et al., 1984 \\
\hline 4 & Xestospongin D & N. exigua (Xestospongia exigua) & Nakagawa et al., 1984 \\
\hline 5 & $(+)$-Araguspongine A & N. exigua (Haliclona exigua) & Venkateswarlu et al., 1994; Venkateswara et al., 1998 \\
\hline 6 & $(+)$-Araguspongine B & $\begin{array}{l}\text { N. exigua (Haliclona exigua) } \\
\text { N. exigua (Xestospongia exigua) }\end{array}$ & $\begin{array}{l}\text { Venkateswarlu et al., 1994; Venkateswara et al., 1998, } \\
\text { Liu et al., } 2004\end{array}$ \\
\hline 7 & $(+)$-Araguspongine $\mathrm{C}$ & N. exigua (Haliclona exigua) & Venkateswarlu et al., 1994; Venkateswara et al., 1998 \\
\hline 8 & (-)-Araguspongine $\mathrm{E}$ & N. exigua (Haliclona exigua) & Venkateswarlu et al., 1994; Venkateswara et al., 1998 \\
\hline 9 & $(+)$-Xestospongin B & N. exigua (Haliclona exigua) & Venkateswarlu et al., 1994; Venkateswara et al., 1998 \\
\hline 10 & (+)-Araguspongine $\mathrm{K}$ & N. exigua (Xestospongia exigua) & Orabi et al., 2002 \\
\hline 11 & $(+)$-Araguspongine $\mathrm{L}$ & N. exigua (Xestospongia exigua) & Orabi et al., 2002 \\
\hline 12 & Araguspongin $\mathrm{C}$ & N. exigua (Haliclona exigua) & Dube et al., 2007 \\
\hline 13 & Xestosin A & N. exigua (Xestospongia exigua) & Iwagawa et al., 2000 \\
\hline 14 & Petrosin & N. similis & Venkateshwar Goud et al., 2003 \\
\hline 15 & Petrosin-A & N. similis & Venkateshwar Goud et al., 2003 \\
\hline 16 & Araguspongine $\mathrm{M}$ & N. exigua (Xestospongia exigua) & Liu et al., 2004 \\
\hline 17 & $\begin{array}{l}\text { 9'-Epi-3ß,3'ß- } \\
\text { dimethylxestospongin } C\end{array}$ & N. exigua & Li et al., 2011 \\
\hline 18 & $\begin{array}{l}\text { 3ß, } 3^{\prime} ß- \\
\text { Dimethylxestospongin C }\end{array}$ & N. exigua & Li et al., 2011 \\
\hline 19 & Demethylxestopongin B & N. exigua & Li et al., 2011 \\
\hline \multicolumn{4}{|c|}{ 3-Alkylpyridine alkaloids } \\
\hline 20 & Renieramycin J & Neopetrosia sp. & Oku et al., 2003 \\
\hline 21 & Renieramycin A & Neopetrosia sp. & Nakao et al., 2004 \\
\hline 22 & Exiguamine A & Neopetrosia exigua & Brastianos et al., 2006 \\
\hline 23 & Njaoamines G & Neopetrosia sp. & Sorek et al., 2007 \\
\hline 24 & Njaoamines $\mathrm{H}$ & Neopetrosia sp. & Sorek et al., 2007 \\
\hline 25 & $\begin{array}{l}\text { 1,2,3,4-tetrahydroquinolin- } \\
\text { 4-one }\end{array}$ & Neopetrosia sp. & Sorek et al., 2007 \\
\hline 26 & Neopetrosiamine A & Neopetrosia proxima & Wei et al., 2010 \\
\hline 27 & Xestoproxamine A & N. proxima & Morinaka and Molinski, 2011 \\
\hline 28 & Xestoproxamine B & N. proxima & Morinaka and Molinski, 2011 \\
\hline 29 & Xestoproxamine C & N. proxima & Morinaka and Molinski, 2011 \\
\hline \multicolumn{4}{|c|}{ Pyridoacridine alkaloids } \\
\hline 30 & $\begin{array}{l}\text { 1-Hydroxydeoxy- } \\
\text { amphimedine }\end{array}$ & N. carbonaria & Wei et al., 2010 \\
\hline 31 & $\begin{array}{l}\text { 3-Hydroxydeoxy- } \\
\text { amphimedine }\end{array}$ & N. carbonaria & Wei et al., 2010 \\
\hline 32 & Debromopetrosamine & N. carbonaria & Wei et al., 2010 \\
\hline
\end{tabular}




\section{Table II}

\section{Chemical constituent of the Neopetrosia genus (Cont.)}

\begin{tabular}{|c|c|c|c|}
\hline No. & Compound class and name & Source & Reference \\
\hline 33 & Amphimedine & N. carbonaria & Wei et al., 2010 \\
\hline 34 & Neoamphimedine & N. carbonaria & Wei et al., 2010 \\
\hline 35 & Deoxyamphimedine & N. carbonaria & Wei et al., 2010 \\
\hline \multicolumn{4}{|c|}{ Others alkaloids } \\
\hline 36 & Motuporamines A & N. exigua (Xestospongia exigua) & Williams et al., 1998; Williams et al., 2002 \\
\hline 37 & Motuporamines B & N. exigua (Xestospongia exigua) & Williams et al., 1998; Williams et al., 2002 \\
\hline 38 & Motuporamines C & N. exigua (Xestospongia exigua) & Williams et al., 1998; Williams et al., 2002 \\
\hline 39 & Motuporamines D & N. exigua (Xestospongia exigua) & Williams et al., 1998; Williams et al., 2002 \\
\hline 40 & Motuporamines E & N. exigua (Xestospongia exigua) & Williams et al., 1998; Williams et al., 2002 \\
\hline 41 & Motuporamines F & N. exigua (Xestospongia exigua) & Williams et al., 1998; Williams et al., 2002 \\
\hline 42 & Motuporamines - a mixture of $\mathrm{G}, \mathrm{H}$, and I & N. exigua (Xestospongia exigua) & Williams et al., 1998; Williams et al., 2002 \\
\hline 43 & 7,8-Dihydrotubastrine & $\begin{array}{l}\text { N. contignata (Petrosia } \\
\text { cf. contignata) }\end{array}$ & Sperry and Crews, 1998 \\
\hline 44 & 4-Deoxy-7,8-dihydrotubastrine & $\begin{array}{l}\text { N. contignata (Petrosia } \\
\text { cf. contignata) }\end{array}$ & Sperry and Crews, 1998 \\
\hline \multicolumn{4}{|c|}{ Quinones } \\
\hline 45 & Tetrahydrohalenaquinone A & N. carbonaria & Alviet al., 1993 \\
\hline 46 & Tetrahydrohalenaquinone B & N. carbonaria & Alvi et al., 1993 \\
\hline 47 & 14-Methoxyhalenaquinone & N. carbonaria & Alvi et al., 1993 \\
\hline 48 & Halenquinone & $\begin{array}{l}\text { N. carbonaria } \\
\text { N. exigua (Xestospongia exigua) }\end{array}$ & Alvi et al., 1993 \\
\hline 49 & Halenquinol & $\begin{array}{l}\text { N. carbonaria } \\
\text { N. sapra } \\
\text { N. seriata (Petrosia seriata) }\end{array}$ & Alvi et al., 1993 \\
\hline 50 & $\begin{array}{l}\text { Halenquinol sulfate } \\
\text { Xestoquinol sulfate }\end{array}$ & $\begin{array}{l}\text { N. carbonaria } \\
\text { N. sapra }\end{array}$ & $\begin{array}{l}\text { Alvi et al., 1993; Kobayashi et al., 1985; } \\
\text { Kobayashi et al., } 1992\end{array}$ \\
\hline 51 & Xestoquinone & N. carbonaria & Alvi et al., 1993 \\
\hline 52 & Xestoquinolide A & N. carbonaria & Alvi et al., 1993 \\
\hline 53 & Xestoquinolide B & N. carbonaria & Alvi et al., 1993 \\
\hline 54 & Xestosaprol A & N. sapra & Kobayashi et al., 1992 \\
\hline 55 & Xestosaprol B & N. sapra & Kobayashi et al., 1992 \\
\hline 56 & Xestosaprol C & N. sapra & Kubota et al., 2008 \\
\hline 57 & Neopetrosiquinone A & N. proxima & Winder et al., 2011 \\
\hline 58 & Neopetrosiquinone B & N. proxima & Winder et al., 2011 \\
\hline 59 & 1,2-Dihydroisoquinoline & N. similis (Petrosia similis) & Ramesh et al., 1999 \\
\hline 60 & Isoquinolinequinone & N. similis (Petrosia similis) & Ramesh et al., 1999 \\
\hline \multicolumn{4}{|c|}{ Sterols } \\
\hline 61 & Galactosyl diacylglycerols & N. exigua (Xestospongia exigua) & Liu et al., 2004 \\
\hline 62 & Galactosyl diacylglycerols & N. exigua (Xestospongia exigua) & Liu et al., 2004 \\
\hline 63 & Galactosyl diacylglycerols & N. exigua (Xestospongia exigua) & Liu et al., 2004 \\
\hline
\end{tabular}


Table II

Chemical constituent of the Neopetrosia genus (Cont.)

\begin{tabular}{|c|c|c|c|}
\hline No. & Compound class and name & Source & Reference \\
\hline 64 & 24-Methyl cholesterol & N. exigua (Xestospongia exigua) & Liu et al., 2004 \\
\hline 65 & 5, 6-Dihydrocholesterol & N. exigua (Xestospongia exigua) & Liu et al., 2004 \\
\hline 66 & $\beta$-Sitosterol & N. exigua (Xestospongia exigua) & Liu et al., 2004; Cerqueira et al., 2003 \\
\hline 67 & $5 a, 8 a-E p i d i o x y$ sterols & N. exigua (Xestospongia exigua) & Liu et al., 2004 \\
\hline 68 & $5 a, 8 a-E p i d i o x y$ sterols & N. exigua (Xestospongia exigua) & Liu et al., 2004 \\
\hline 69 & $5 a, 8 a-E p i d i o x y$ sterols & N. exigua (Xestospongia exigua) & Liu et al., 2004 \\
\hline 70 & $\begin{array}{l}\text { 5a, 8a-Epidioxy-24a- } \\
\text { ethylcholest-6-en-3-ol }\end{array}$ & N. exigua (Xestospongia exigua) & Cerqueira et al., 2003 \\
\hline 71 & Clionasterol & N. exigua (Xestospongia exigua) & Cerqueira et al., 2003 \\
\hline 72 & Clionasterol monoacetate & N. exigua (Xestospongia exigua) & Cerqueira et al., 2003 \\
\hline 73 & Xestobergsterol A & N. contignata (Petrosia cf. contignata) & Sperry and Crews, 1998 \\
\hline \multicolumn{4}{|c|}{ Terpenoids } \\
\hline 74 & Xestovanin A & N. vanilla (X. vanilla) & Northcote and Andersen, 1989 \\
\hline 75 & Secoxestovanin A & N. vanilla (X. vanilla) & Northcote and Andersen, 1989 \\
\hline 76 & Isoxestovanin A & N. vanilla (Xestospongia vanilla) & Morris et al., 1991 \\
\hline 77 & Xestovanins B & N. vanilla (Xestospongia vanilla) & Morris et al., 1991 \\
\hline 78 & Xestovanins C & N. vanilla (Xestospongia vanilla) & Morris et al., 1991 \\
\hline 79 & Dehydroxestovanin C & N. vanilla (Xestospongia vanilla) & Morris et al., 1991 \\
\hline 80 & Xestodiol & N. vanilla & Northcote and Andersen, 1989; Morris et al., 1991 \\
\hline 81 & Xestenone & N. vanilla & Northcote and Andersen, 1989 \\
\hline 82 & Xestolide & N. vanilla & Morris et al., 1991 \\
\hline 83 & Secoxestenone & N. vanilla & Northcote and Andersen, 1989 \\
\hline 84 & Secodehydroxestovanine A & N. vanilla & Morris et al., 1991 \\
\hline \multicolumn{4}{|c|}{ Peptides } \\
\hline 85 & Neopetrosiamdes A and B & Neopetrosia sp. & Williams et al., 2005; Towle et al., 2013 \\
\hline
\end{tabular}

njaoamines $\mathrm{G}(23)$ and $\mathrm{H}(\mathbf{2 4})$ and 1,2,3,4-tetrahydroquinolin-4-one (25) were obtained from the sponge Neopetrosia sp. collected from Pemba Island, Tanzania (Sorek et al., 2007). A pentacyclic hydroquinone exiguaquinol was isolated from the methanol extract of the Australian sponge Neopetrosia exigua (Leone et al., 2008). Neopetrosiamine A (26) was extracted from the marine sponge Neopetrosia proxima collected off the west coast of Puerto Rico (Wei et al., 2010). Three xestoproxamines A (27), B (28) and C (29) were isolated from the Bahamian sponge $N$. proxima(Morinaka and Molinski, 2011).

\section{Pyridoacridine alkaloids}

Six pyridoacridine alkaloids have been reported from $N$. carbonaria collected from Palau including 1-hydroxydeoxyamphimedine (30), 3-hydroxy-deoxyamphimedine (31), debromopetrosamine (32), amphimedine (33), neoamphimedine (34) and deoxyamphimedine (35) (Wei et al., 2010).

\section{Others alkaloids}

Eight motuporamines including motuporamine A (36), $\mathrm{B}(37)$ and $C(38), \mathrm{D}(39), \mathrm{E}(40)$ and $\mathrm{F}(41)$ and a mixture of G, H, and I (42) were obtained from N. exigua (Xestospongia exigua) collected from Papua New Guinea yielded (Williams et al., 1998; Williams et al., 2002). Two phenethyl-guanidine derivatives, 7,8-dihydrotubastrine (43) and 4-deoxy-7,8-dihydrotubastrine (44), were isolated from the from the Indo-Pacific sponge $N$. contignata (Petrosia cf. contignata) (Sperry and Crews, 1998).

\section{Quinones}

More than 21 quinone and hydroquinone derivatives have been isolated from Neopetrosia genus. Several 
soluble fraction of N. exigua (Haliclona exigua) (Dube et al., 2007). A bis-quinolizidine alkaloid, xestosin A (13), was isolated from the Papua New Guinean sponge $N$. exigua (Xestospongia exigua) (Iwagawa et al., 2000). Two bis-quinolizidine alkaloids namely, petrosin (14) and petrosin-A (15) were isolated from $N$. similis (Venkateshwar Goud et al., 2003). Three macrocyclic quinolizidines alkaloids were obtained from the nbutanol extract of $N$. exigua (Xestospongia exigua) collected in Palau including araguspongine M (16), araguspongines B (6) and D (1) (In 2004, Liu et al., 2004). 9'-Epi-3ß,3' $\$$-dimethylxestospongin C (17), xestospongin A (1), 3ß, $3^{\prime} \beta$-dimethylxestospongin C (18) and demethylxestopongin B (19) were isolated from the Hainan sponge N. exigua (Li et al., 2011).

\section{3-Alkylpyridine alkaloids}

Ten 3-alkylpyridine alkaloids were reported from Neopetrosia genus. Renieramycin J (20), a tetrahydroisoquinoline alkaloid, was reported from Neopetrosia sp. collected from Iwo-Jima Island, Japan (Oku et al., 2003). Renieramycin A (21) was reported from the Japanese sponge Neopetrosia sp. (Nakao et al., 2004). A hexacyclic alkaloid, exiguamine A (22), was isolated from Neopetrosia exigua collected in Papua New Guinea (Brastianos et al., 2006). Two polycyclic alkaloids,
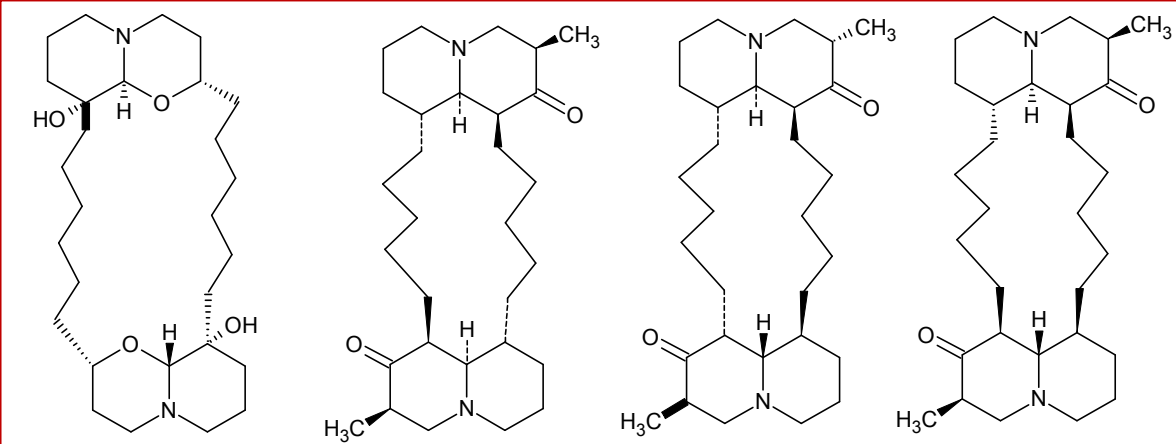

Xestosin A (13)

Petrosin (14)

Petrosin-A (15) Araguspongine M (16)

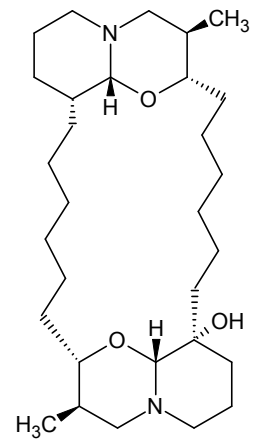

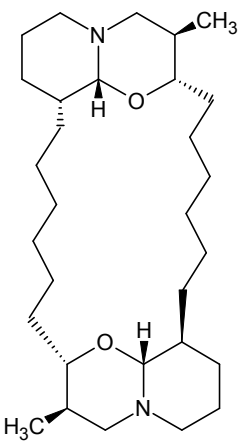

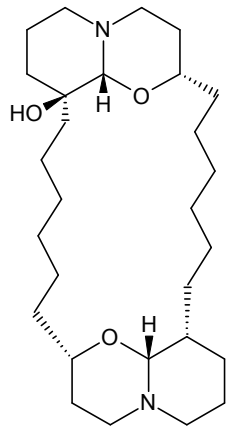

9'-epi-3beta,3'beta-

3beta, 3 'beta-

Dimethylxestospongin B (19)

Dimethylxestospongin C (17) Dimethylxestospongin C (18)

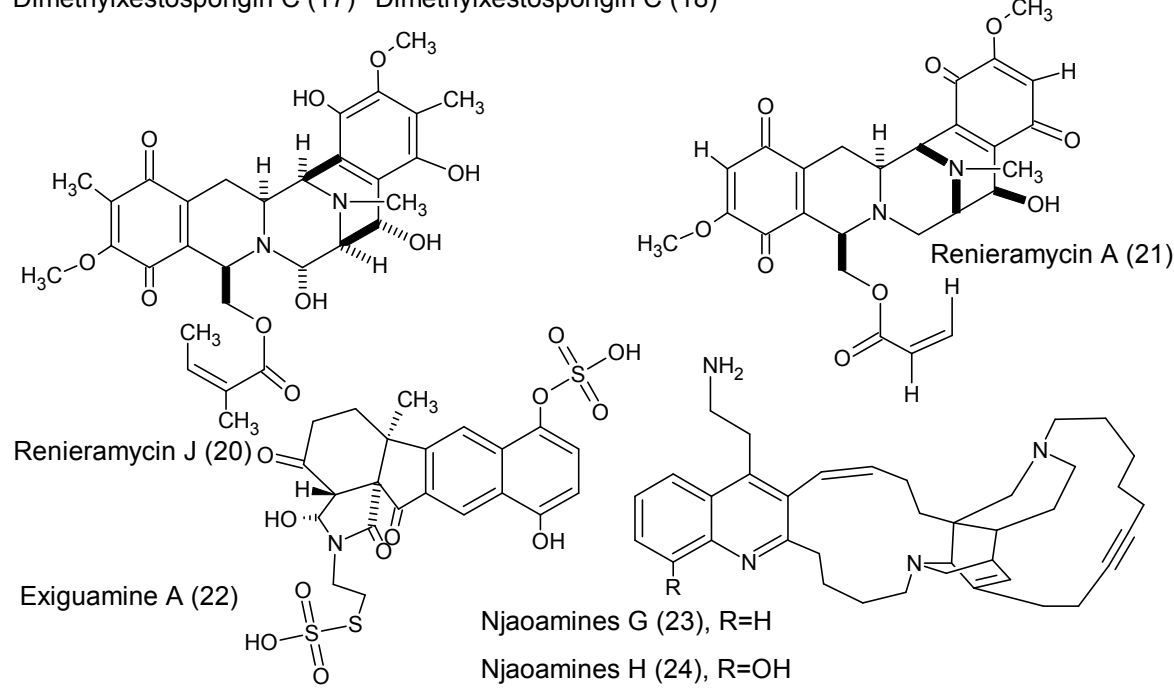




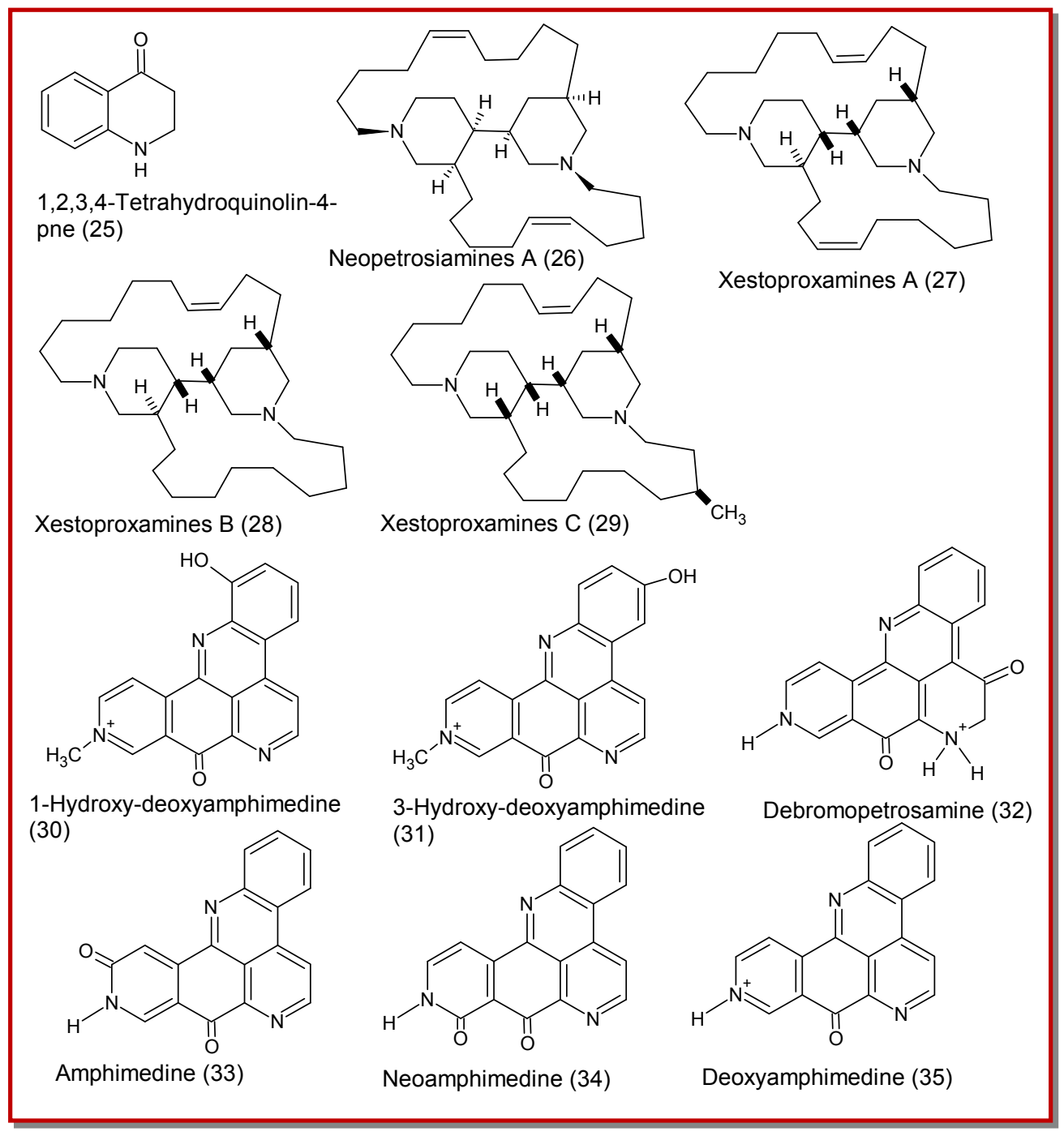

polycyclic quinones and hydroquinones compounds were isolated from $N$. Carbonaria including tetrahydrohalenaquinone A (45), tetrahydrohalenaquinone B (46), 14-methoxyhalenaquinone (47), halenquinone (48), halenquinol (49), halenquinol sulfate (50), xestoquinone (51), xestoquinolide A (52) and xestoquinolide B (53) (Alvi et al., 1993). Halenaquinone (48) was obtained from N. exigua (Xestospongia exigua) benzene extract (Roll et al., 1983). Halenaquinol (49) was reported from N. Sapra (Kobayashi et al., 1985) and N. seriata (Petrosia seriata) (Gorshkova et al., 1999). Halenaquinol sulfate (also called xestoquinol sulfate) (50) was isolated from Okinawan sponge N. Sapra (Kobayashi et al., 1985; Kobayashi et al., 1992). Two other hydroquinones were isolated from Okinawan marine sponge $N$. sapra namely xestosaprols A (54) and B (55) (Kubota et al., 2008). Xestosaprol C (56), a pentacyclic hydroquinone sulfate, was obtained from an Okinawan marine sponge $N$. sapra (Kubota et al, 2008). Two sesquiterpene benzoquinones neopetrosiquinones A (57) and B (58), were reported from the ethanol extract of N. Proxima
(Winder et al., 2011). 1, 2-dihydroisoquinoline (59) and isoquinoline-quinone (60) were obtained from the sponge N. similis (Petrosia similis) (Ramesh et al., 1999).

\section{Sterols}

14 sterols compounds were isolated from Neopetrosia genus. Seven sterols derivatives were obtained from the n-butanol extract of N. exigua (Xestospongia exigua) collected in Palau including three galactosyl diacylglycerols $(61,62,63)$, 24-methyl cholesterol (64), 5, 6 -dihydrocholesterol (65), $\beta$-sitosterol (66), and three $5 a$, $8 a-e p i d i o x y$ sterols $(67,68,69) .5 a, 8 a-e p i d i o x y ~ s t e r o l$, $5 a, 8 a-e p i d i o x y-24 a-e t h y l c h o l e s t-6-e n-3-o l$ (70), and clionasterol (71), clionasterol monoacetate (72) and $\beta$ sitosterol (66) were reported from $N$. exigua (Xestospongia exigua) (Cerqueira et al., 2003). The sterol xestobergsterol A (73), was isolated from N. contignata (Petrosia cf. contignata) (Sperry and Crews, 1998).

\section{Terpenoids}

Twelve terpenoids were found in Neopetrosia genus. 


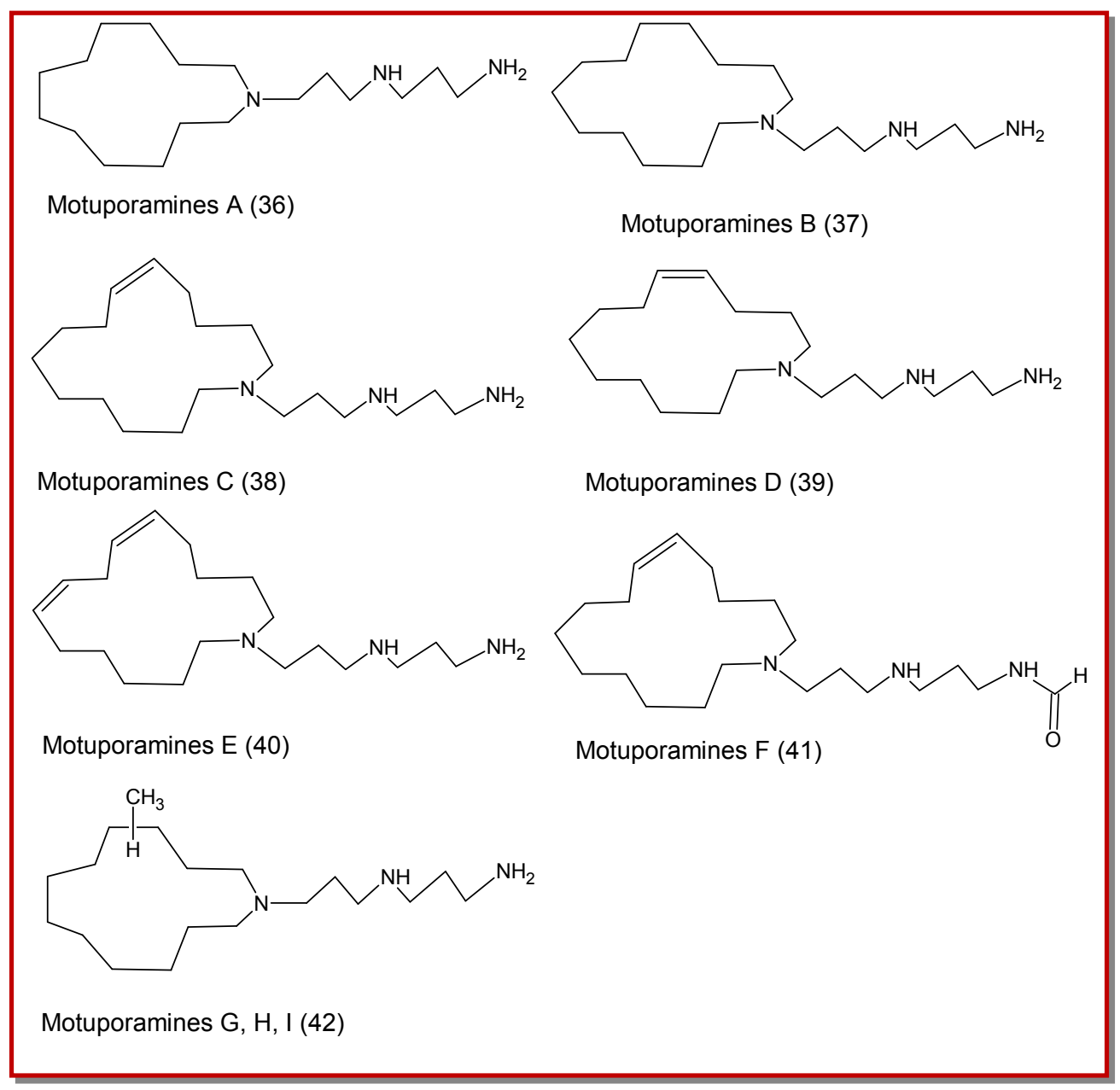

Two triterpene glycosides, xestovanin A (74) and secoxestovanin A (75) were reported from the Northeastern Pacific sponge $N$. vanilla (X. vanilla) (Northcote and Andersen, 1989; Andersen et al., 1988). Isoxestovanin A (76), Xestovanins B (77), C (78) and dehydroxestovanin C (79) were obtained from the Northeastern Pacific species of N. vanilla (Xestospongia vanilla) (Morriset al., 1991). Xestodiol (80), Xestenone (81), Xestolide (82), secoxestenone (83), and secodehydroxestovanine A (84) were isolated from $N$. Vanilla (Northcote and Andersen, 1987; Northcote and Andersen, 1989; Morriset al., 1991).

\section{Peptides}

Only two diastereomeric tricyclic peptides, neopetrosiamdes A and B (85), which differ only by the stereochemistry of the sulfoxide group, were isolated from Neopetrosia sp. collected in Papua New Guinea (Williams et al., 2005; Towle et al., 2013).

\section{Biological activities}

\section{Antimicrobial, antifouling and anti-HIV activities}

The in vitro antimicrobial and antifouling activities of
Neopetrosia extracts have been confirmed. In screening of invertebrate materials for antifouling activity, MoraCristancho and co-authors (2011) identified the $\mathrm{CH} 2 \mathrm{Cl} 2 / \mathrm{MeOH}$ extract of N.carbonaria as a potent antimicrobial extract against the fouling bacterial strains Oceanobacillus iheyensis, Kocuria sp., Vibrio harveyi and Bacillus megaterium with more than $12 \mathrm{~mm}$ inhibition zone (300 $\mu \mathrm{g}$ extract concentration) (MoraCristancho et al., 2011). Aqueous and organic extracts from $N$. exigua exhibited stronger antibacterial and antifungal activities. The highest activity was obtained for the aqueous extract against the Gram-positive bacteria B. cereus (inhibition zone $25 \mathrm{~mm}$ and MIC 0.07 $\mathrm{mg} / \mathrm{mL})$ and $S$. aureus $(17.5 \mathrm{~mm}$ and $0.12 \mathrm{mg} / \mathrm{mL})$ and against C. albicans (21 $\mathrm{mm}$ and $0.32 \mathrm{mg} / \mathrm{mL}$ ) (Qaralleh et al., 2010; Majali et al., 2015). The methanol extract of the marine sponge $N$. exigua (Haliclona exigua) was tested in micro-dilution method and indicated significant antifungal activity in vitro against Candida albicans (MIC $=7.8 \mu \mathrm{g} / \mathrm{mL}$ ), Cryptococcus neoformans $(\mathrm{MIC}=31.2 \mu \mathrm{g} / \mathrm{mL})$, Sporothrix schenckii $(\mathrm{MIC}=31.2$ $\mu \mathrm{g} / \mathrm{mL}$ ), Trichophyton mentagrophytes (MIC $=31.2 \mu \mathrm{g}$ / $\mathrm{mL}$ ), Aspergillus fumigatus (MIC $=31.2 \mu \mathrm{g} / \mathrm{mL}$ ) and Candida parapsilosis (MIC $=7.8 \mu \mathrm{g} / \mathrm{mL}$ ) (Lakshmi et al., 2010). The extract provided one active compound 


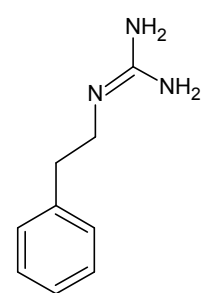<smiles>NC(N)=NCCc1cccc(O)c1</smiles><smiles>O=C1CCC(O)c2cc3c(cc21)[C@H]1CCC(O)[C@H]2COC=C1C32</smiles>

7,8-Dihydrotubastrine (43) 4-Deoxy-7,8-dihydrotubastrine (44) Tetrahydrohalenquinone A (45)<smiles>O=C1C2=C3[C@@H](CCC(O)[C@@H]3C2)c2cc3c(cc21)C(=O)CC[C@H]3O</smiles><smiles>COC1=CC(=O)c2cc3c(cc2C1=O)C(=O)c1occ2c1[C@H]3CCC2=O</smiles><smiles>O=C1C=CC(=O)c2cc3c(cc21)C(=O)c1occ2c1[C@@H]3CCC2=O</smiles>

Tetrahydrohalenquinone B (46) 14-Methoxyhalenquinone (47)

Halenquinone (48)<smiles>[R]c1ccc(O)c2cc3c(cc12)[C@H]1CCC(=O)c2coc(c21)C3=O</smiles><smiles>O=C1C=CC(=O)c2cc3c(cc21)C(=O)c1occ2c1[C@@H]3CCC2</smiles><smiles>CC1=CCC[C@@]2(C)C1=COC(=O)c1cc3c(cc12)C(=O)C=CC3=O</smiles>

Halenquinol (49), $\mathrm{R}=\mathrm{OH}$

Xestoquinone (51), $\mathrm{R}=\mathrm{H}$

Xestoquinolide A (52)

Halenquinol sulfate (50), $\mathrm{R}=\mathrm{SO}_{3} \mathrm{Na}$

or Xestoquinol sulfate<smiles>[Y]C1=C2C(=O)c3cc4c(cc3C(=O)C2=[Y7]CC1)[C@@]1(C)CCC=C(C)C1=COC4=O</smiles><smiles>[R]C1CCC([R])c2cc3c(cc21)C(=O)C1=C3[C@]2(C)CC[C@@H](O)[C@H]2C1</smiles>

Xestosaprols A (54), R1=H,OH R2=O<smiles>C[C@]12CCC(=O)C3=C1[C@@](O)(O[C@H](O)C3=O)c1cc3c(O[R](=O)(O)O)ccc(O)c3cc12</smiles>

Xestoquinolide B (53) Xestosaprols B (55), R1=R2=H,OH

$\mathrm{X}=\mathrm{NH}, \mathrm{Y}=\mathrm{SO} 2$ or $\mathrm{X}=\mathrm{SO} 2, \mathrm{Y}=\mathrm{NH}$

namely araguspongin C (7), that showed promising activity against Cryptococcus neoformans, Sporothrix schenckii, Trichophyton mentagrophytes and Aspergillus fumigatus with identical MIC of $50 \mu \mathrm{g} / \mathrm{mL}$. In another study, araguspongin C (7) isolated from $N$. exigua exhibited potent antifouling activity with EC50 $=6.6$ $\mu \mathrm{g} / \mathrm{mL}$ and low toxicity with LC50 $=18 \mu \mathrm{g} / \mathrm{mL}$ (Limna Mol et al., 2009; Limna Mol et al., 2010).

In a screening of crude extracts of 6 species of sponges for their antifouling activity, Limna Mol and co-authors (2010)reported the methanol/acetone extract of the $N$. exigua as a moderate antifouling extract. $N$. exigua extract exhibited moderate antibacterial activity against the fouling bacterial strains; Bacillus cereus; B. pumilus; B. megaterium; Pseudoalteromonas haloplanktis;
Pseudomonas chlororaphis; P. putida; P. aeruginosa. In a preliminary screening study, the chloroform and methanol extracts of $N$. proxima collected from the Uraba Gulf in the Colombian Caribbean region, showed no antibacterial activity against Staphylococcus aureus ATCC 25923 and Escherichia coli ATCC 25922 and antifungal activity against Candida albicans ATCC 10231 (Galeano and Martínez, 2007). In contrast, the organic extract obtained from $N$. proxima showed in vitro antibacterial activity against the Gram-positive Staphylococcus aureus and Streptococcus faecalis and antifungal activity against Candida albicans (Mora et al., 2008).

A pentacyclic polyketide, halenaquinone (48) isolated from the benzene extract of $N$. exigua (Xestospongia 
<smiles>O=C[C@H]1CCC[C@@H]2c3cc4c(cc3C(=O)C[C@H]12)C(=O)C=CC4=O</smiles>

Neopetrosiquinones A (57) Neopetrosiquinones B (58)<smiles>COc1cc(O)c2c(c1)C(CC(=O)O)N(C=O)C=C2</smiles>

1,2-Dihydroisoquinoline (59) Isoquinolinequinone (60)

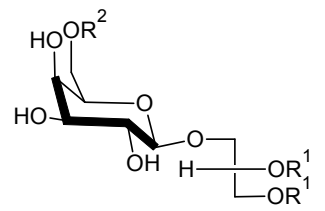

Galactosyl diacylglycerol

$\mathrm{R} 1=16: 0116: 1 \mathrm{R} 2=\mathrm{H}(61)$

$\mathrm{R} 1=16: 0118: 2 \mathrm{R} 2=\mathrm{H}(62)$

$\mathrm{R} 1=16: 0 \backslash 16: 1 \mathrm{R} 2=$ alpha-D-galactopyranosyl (63)<smiles>CC(C)CCCC(C)C1CCC2C3CCC4CC(O)CCC4(C)C3CCC12C</smiles>

5,6-Dihydroxycholestrol (65)

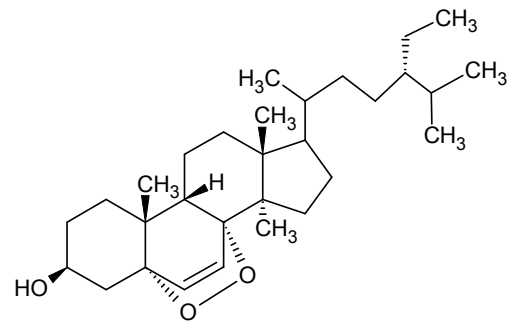

5Alpha,8alpha-epidioxy sterol (67)

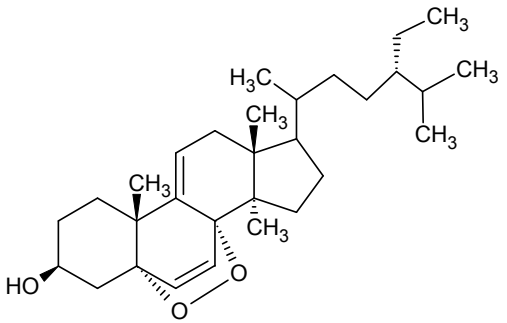

5Alpha,8alpha-epidioxy sterol (69)<smiles>CC(C)C(C)CCC(C)C1CCC2C3CC=C4CC(O)CCC4(C)C3CCC12C</smiles>

24-Methylcholestrol (64)<smiles>CCC(CCC(C)C1CCC2C3CC=C4CC(O)CCC4(C)C3CCC12C)C(C)C</smiles>

Beta-sitosterol (66)

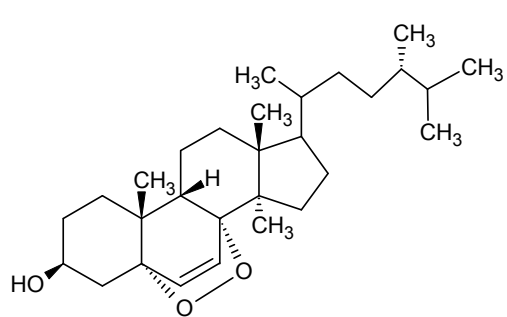

5Alpha,8alpha-epidioxy sterol (68)

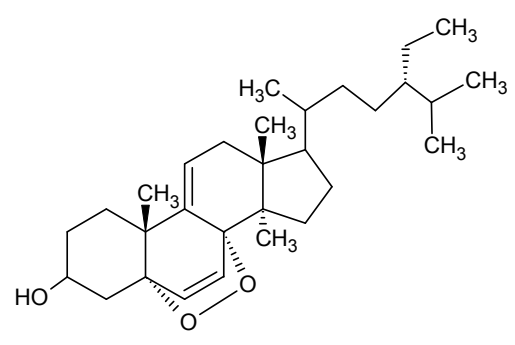

5Alpha,8alpha-epidioxy-24alpha -ethylcholestrol-6-en-3-ol (70) 


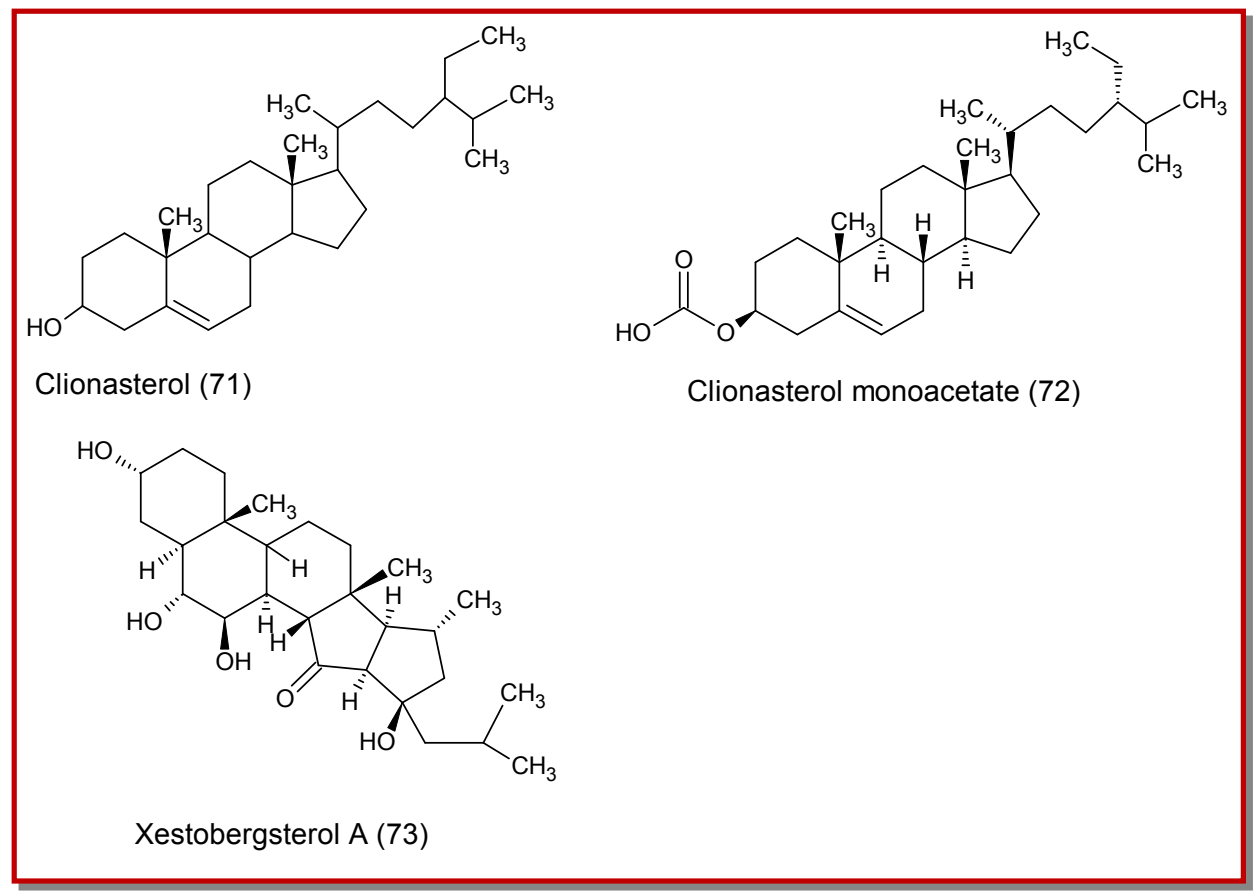

exigua) was reported with antibacterial activity against Staphylococcus aureus and Bacillus subtilis (Roll et al., 1983). (+)-Araguspongine C (7) was reported with antituberculosis activity with MIC $3.9 \mu \mathrm{M}$ (Orabi et al., 2002). The anti-tuberculosis activity was confirmed for neopetrosiamine A (26) in vitro against a pathogenic strain of Mycobacterium tuberculosis (H37Rv) with MIC value of $7.5 \mu \mathrm{g} / \mathrm{mL}$ (Weiet al., 2010). A pentacyclic hydroquinone exiguaquinol inhibited Helicobacter pylori glutamate racemase (MurI) with an $\mathrm{IC}_{50}$ of $4.4 \mu \mathrm{M}$. The triterpene glycosides, xestovanin A was reported from $N$. vanilla with antifungal activity against Phytium ultimum (Northcote and Andersen, 1989).

Two bis-quinolizidine alkaloids namely, petrosin (14) and petrosin-A (15) were reported as anti-HIV inhibitors with $\mathrm{IC}_{50}$ values of 41.3 and $52.9 \mu \mathrm{m}$, respectively (Venkateshwar et al., 2003).

Cytotoxic, antitumor, anti-proliferation, anti-angiogenic and anti-invasion activities

Selective cytotoxic activity was indicated for $N$. contignata extract against tumor cell lines HT-29, T47D and Casky with $\mathrm{IC}_{50}$ of $78.9,35.6$ and $36.2 \mu \mathrm{g} / \mathrm{mL}$, respectively (Abdillah et al., 2013a). Using BST test, the hydro-ethanolic extract of $N$. contignata and $N$. exigua (X. exigua) exhibited strong toxicity with $\mathrm{LC}_{50}$ equal to 155 and 547 ppm, respectively.

The pyridoacridine alkaloids, amphimedine (33) isolated from $N$. carbonaria exhibited potent cytotoxic activity that caused a phenotype in zebra fish embryos at $30 \mu \mathrm{M}$ (Wei et al., 2010).

In 2004, Liu and colleagues (2004) reported the cytotoxic activities of araguspongine M (16), araguspongines B (6) and D (1) and three 5a, 8aepidioxy sterols (67-69) against the human leukemia cell line HL-60 with $\mathrm{IC}_{50}$ values of 5.5, 5.5, 5.9, 22.4, 9.5, and $9.6 \mu \mathrm{M}$, respectively. Renieramycin A (21) obtained from Neopetrosia sp. exhibited cytotoxicity with $\mathrm{IC}_{50}=$ $2.2 \mu \mathrm{g} / \mathrm{mL}$. Renieramycin J (20) was reported with cytotoxic activity against 3 Y1, HeLa, and P388 cells with $\mathrm{IC}_{50}$ of $5.3,12.3$, and $0.53 \mathrm{nM}$, respectively (Oku et al., 2003). High concentration of renieramycin J induced morphological changes in 3Y1 cells in which these changes might be refer to RNA and/or protein synthesis inhibition. Sorek and co-authors (2007) reported that njaoamines $G(23)$ and $H(24)$ possess potent brine shrimp toxicity with $\mathrm{LD}_{50}$ values of 0.17 and $0.08 \mu \mathrm{g} / \mathrm{mL}$, respectively. Demethylxestopongin B (19) was isolated from the Hainan sponge N. exigua as a potent cytotoxic compound against human tumor cell line A-549 with inhibition ratio of $94.3 \%$ at $10 \mu \mathrm{M}$ (Liet al., 2011).

Halenaquinone (48) was found to exhibit anticancer activity through apoptosis. Fujiwara and co-authors (2001) reported that the mechanism of halenaquinoneinduced apoptosis may be explained by the inhibition of phosphatidylinositol 3-kinase activity.

Winder and colleagues (2011) reported the antiproliferation activity of neopetrosiquinones A (57) and B (58) against the DLD-1 human colorectal adenocarcinoma cell line with IC50 values of 3.7 and 9.8 $\mu \mathrm{M}$, respectively and the PANC-1 human pancreatic carcinoma cell line with $\mathrm{IC}_{50}$ values of 6.1 and $13.8 \mu \mathrm{M}$, respectively. Neopetrosiquinone A (57) also inhibited the in vitro proliferation of the AsPC-1 human pancreatic carcinoma cell line with an $\mathrm{IC}_{50}$ value of $6.1 \mu \mathrm{M}$. 

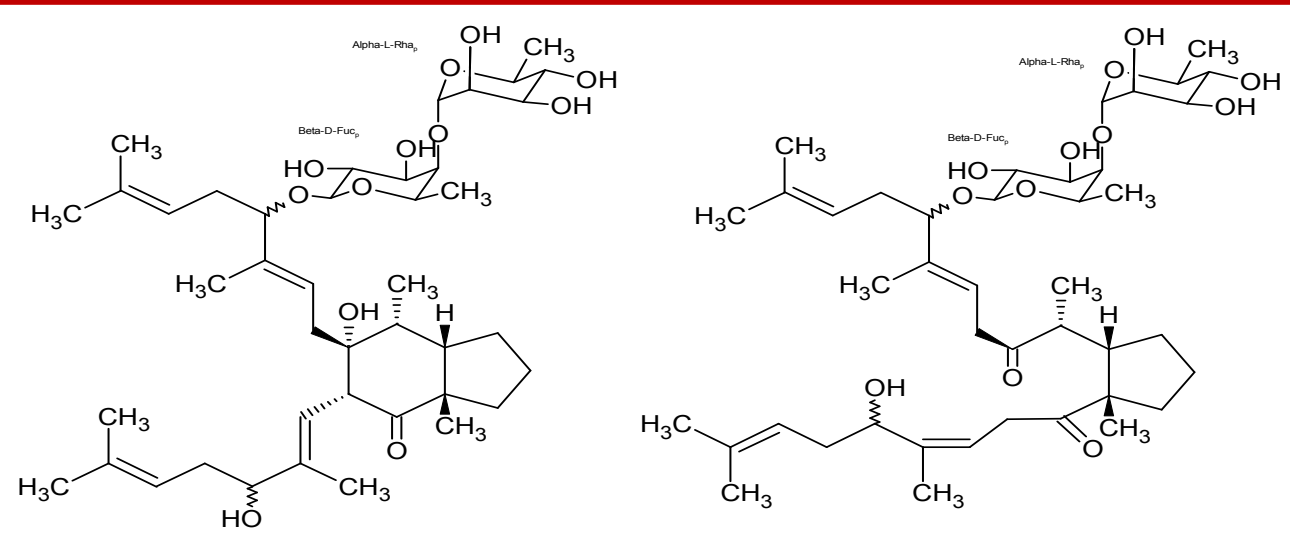

Xestovanin A (74)
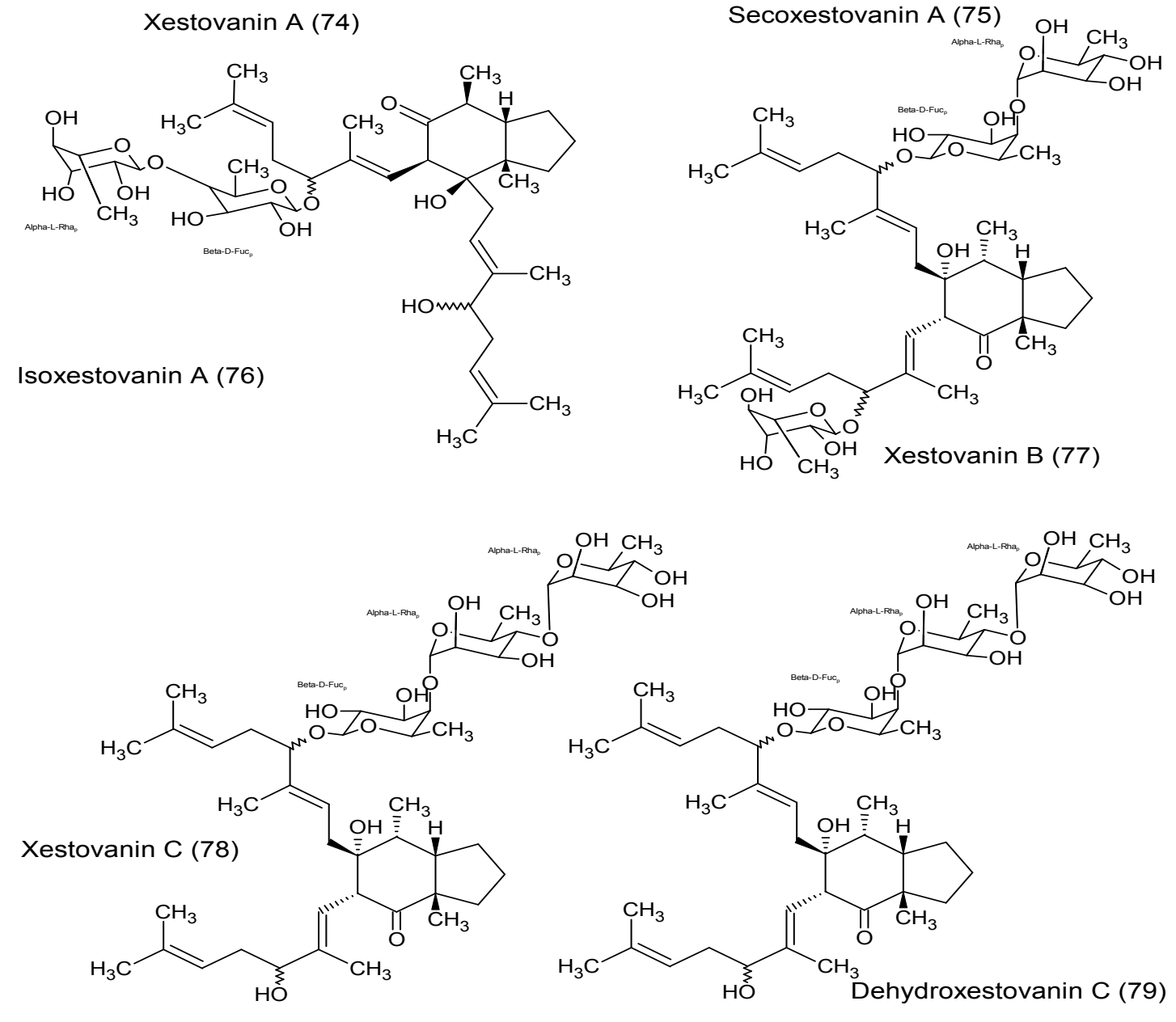

In vitro anti-tumor screening showed that neopetrosiamine A (26) exhibited strong inhibitory activity against MALME-3M melanoma cancer, CCRFCEM leukemia and MCF7 breast cancer with IC values of 1.5, 2.0 , and $3.5 \mu \mathrm{M}$, respectively. Notably, neopetrosiamine A did not exhibit cytotoxicity against VERO cells $\left(\mathrm{IC}_{50}=\right.$ $42.4 \mu \mathrm{g} / \mathrm{mL})$.

Motuporamines A (36), B (37) and C (38) and the mixture of G, H and I (42) exhibited anti-invasion activity. In 2001, Roskelley and colleagues (2001) showed that the compound motuporamine C (38) interferes with the migration of human breast carcinoma, prostate carcinoma and glioma cells in culture and inhibited angiogenesis in both an in vitro sprouting assay and an in vivo chick chorioallantoic membrane assay (Williams et al., 1998; Roskelley et al., 2001; Williams et al., 2002).

Neopetrosiamdes A and B (85) were reported as potential anti-metastatic agents that inhibit tumour cell invasion by both amoeboid and mesenchymal 


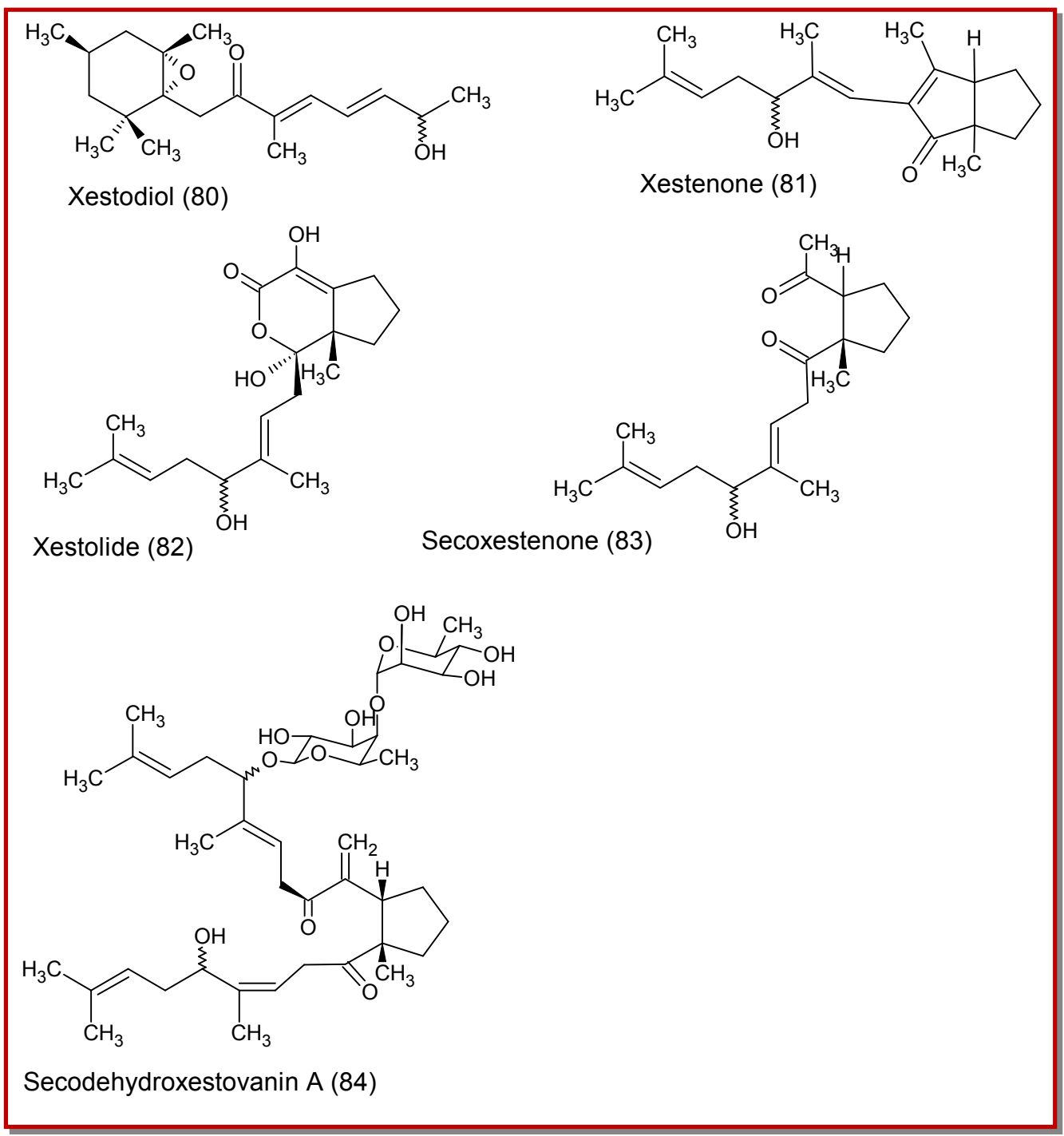

migration pathways (Williams et al., 2005; Towle et al., 2013).

\section{Anti-oxidant activity}

Anti-oxidant activities of $N$. contignata and $N$. exigua (Xestospongia exigua) extract were reported. The hydroethanolic extract of $N$. contignata and $N$. exigua exhibited moderate antioxidant activity with $\mathrm{IC}_{50}<100 \mu \mathrm{g} / \mathrm{mL}$ using DPPH method (Abdillah et al., 2013a).

\section{Enzymes inhibitors}

Exiguamine A (22), has been found to be one of the most potent inhibitor of indoleamine-2, 3-dioxygenase (IDO) in vitro. IDO inhibition can delay tumor growth (Brastianos et al., 2006).

Halenaquinone (48) and 14-methoxyhalenaquinone (47) were reported as a potent protein tyrosine kinase (PTK) inhibitors with $\mathrm{IC}_{50}$ values $<10$ muM (Alvi et al., 1993). This enzyme is associated with proliferative disease such as cancer.
Halenaquinol (49), isolated from $N$. seriata, was reported as inhibitor for rat brain cortex $\mathrm{Na}^{+}, \mathrm{K}^{+}$ATPase with an $I_{50}$ value of $1.3 \times 10-6 \mathrm{M}$ or $325 \mathrm{nmol}$ per mg of protein (Gorshkova et al., 1999). Further investigation suggested that halenaquinol interacts with the essential sulfhydryls in or near the ATP-binding site of $\mathrm{Na}+, \mathrm{K}+-\mathrm{ATPase}$. This interaction resulted in a change of protein conformation and subsequent alteration of overall and partial enzymatic reactions (Gorshkova et al., 2001).

Araguspongines A (5) and C (7) showed an ability to inhibit rat brain nitric oxide synthase activity in vitro with an estimated $\mathrm{IC}_{50}$ of 31.5 and $46.5 \mathrm{mM}$ respectively (Venkateswara et al., 1998).

Araguspongines A (5) and C (7) and xestospongin B (2) were reported as a potent inhibitors for inositol 1, 4, 5triphosphate receptor mediated $\mathrm{Ca}^{2+}$ release and endoplasmic reticulum-calcium pump (Gafni et al., 1997; De Smet et al., 1999). 


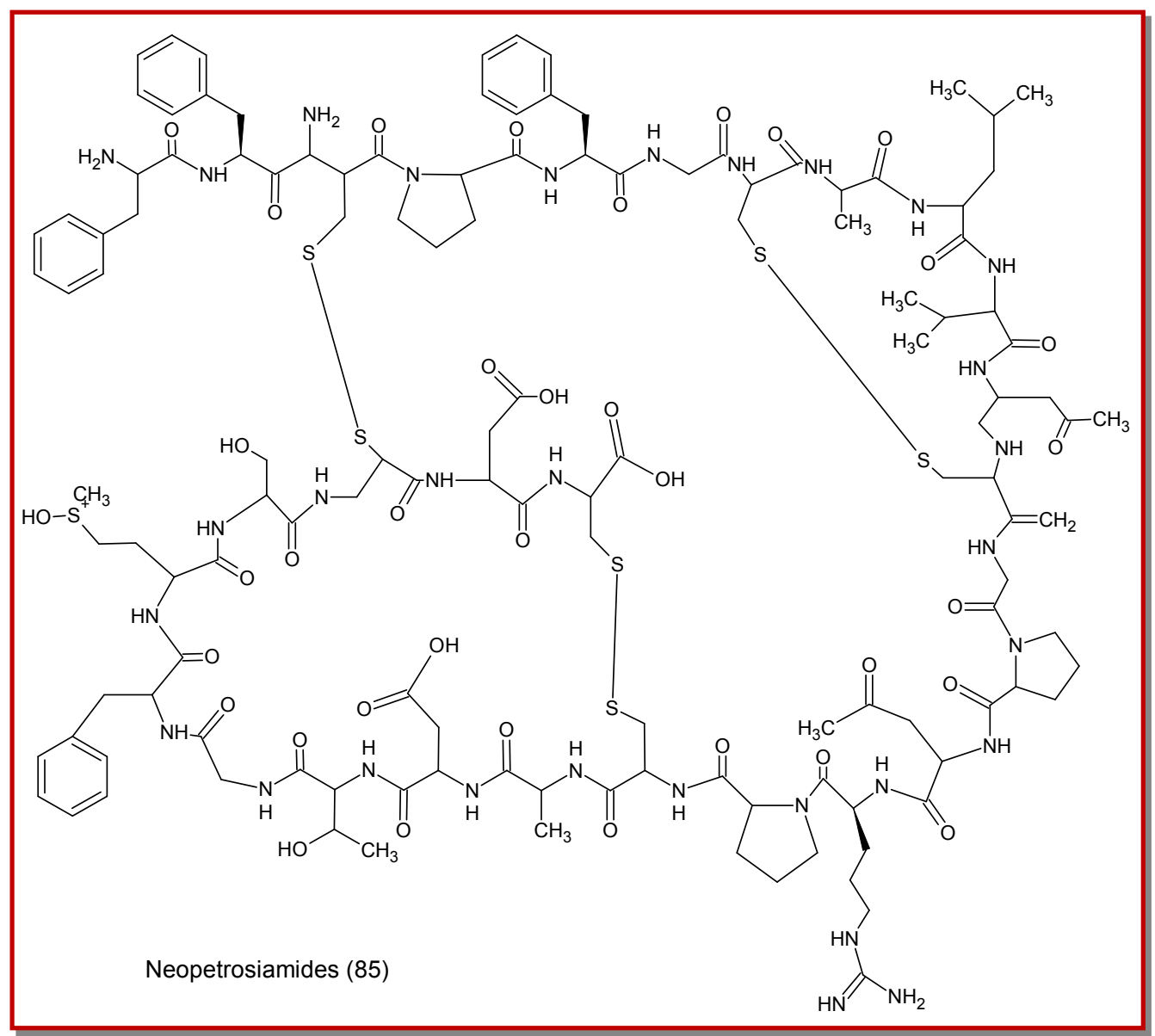

\section{Antiprotozoal activity}

Recently, the anti-malarial activity of $N$. exigua has been reported. Ethanol soluble extracts of $N$. exigua with doses of 400 and $200 \mathrm{mg} / \mathrm{kg}$ showed suppression of growth activity against Plasmodium berghei by $80.7 \%$ and $60.6 \%$, respectively (Abdillah et al., 2013a).

Neopetrosamine A (26) was reported with antiplasmodial activity against Plasmodium falciparum with an $\mathrm{IC}_{50}$ value of $2.3 \mu \mathrm{M}$ (Wei et al., 2010). Renieramycin A (21) exhibited anti-protozoal activity against Leishmania amazonensis with $\mathrm{IC}_{50}=0.2 \mu \mathrm{g} / \mathrm{mL}$ and cytotoxicity with $\mathrm{IC}_{50}=2.2 \mu \mathrm{g} / \mathrm{mL}$ (Nakao et al., 2004). Araguspongine C (7) exhibited in vitro anti-malarial activity against Plasmodium falciparum with $\mathrm{IC}_{50}$ ranged from 280 to $670 \mathrm{ng} / \mathrm{mL}$ (Orabi et al., 2002).

\section{Anti-inflammatory activity and anti-complementary inhibitor}

The anti-inflammatory activity of $N$. proxima and $N$. rosariensis collected from the Colombian Caribbean has been confirmed. The methanolic extract and the different polarity fractions of N. Proxima exhibited in vitro and in vivo anti-inflammatory activities. Total extracts of $N$. proxima $(100 \mathrm{mg} / \mathrm{Kg})$ significantly inhibited the paw edema of rats $(60 \%)$. Dichloro- methane and methanol fractions reduced myeloperoxidase activity (MPO) while there was no significant reduction for the nitric oxide (NO), prostaglandin E2 (PGE2) and tumor necrosis factor alpha (TNF- alpha) (Franco et al., 2012). Total extracts of $N$. rosariensis $(100 \mathrm{mg} / \mathrm{Kg})$ significantly inhibited the paw edema of rats $(72 \%)$. Dichloromethane and methanol fractions reduced myeloperoxidase activity (MPO). Only, dichloromethane fraction of N. rosariensis significantly inhibited nitric oxide (NO) (66\%), prostaglandin E2 (PGE2) (30.5\%) and tumor necrosis factor alpha (TNF-alpha) production (72\%) (Franco et al., 2012). Clionasterol (71), isolated from N. exigua (Xestospongia exigua), exhibited potent anticomplementary inhibitor with $\mathrm{IC}_{50}=4.1 \mu \mathrm{M}$ (Cerqueira et al., 2003).

\section{Other}

Xestospongin A (1), B (2), C (3) and D (4) were found to be active as a vasodilator compounds since they induce relaxation of blood vessel in vivo (Zhou et al., 2010). Halenaquinol (49) was reported from $N$. seriata with a cardioactivity (Gorshkova et al., 1999). Xestosaprol C (56) was reported with cardiotonic activity (Nakamura et al., 1985). Halenaquinone (48), was found to be as an inhibitor of osteoclastogenic differentiation of murine 
RAW264 cells (Tsukamoto et al., 2014).

\section{Chemotaxonomic significance}

A literature search showed that only 9 species out of 27 of Neopetrosia that have been chemically investigated. In general, these species produced alkaloids, quinones, sterols and terpenoids. Macrocyclic quinolizidines are a major kind of metabolite that existed in this genus and more specifically in N. exigua and N. similis. Most other similar macrocyclic quinolizidines $[(+)$-araguspongine A-J] were reported from Xestospongia sp. that has been identified to the genus level(Kobayashi et al., 1989). In this study, 3-alkylpyridine alkaloids were found in $N$. exigua, N. proxima and Neopetrosia sp. The occurrence of 3 -alkylpyridine alkaloids has been reported from other sponge genera including xestospongia, amphimedon and Topsentia suggested that these genera share similar biosynthetic pathways. Previous studies reported that Xestospongia wiedenmayeri and X. ingens contain 3alkylpyridine alkaloids such as xestamine and ingamine, respectively (Quirion et al., 1992; Kong and Andersen, 1995; Takekawa et al., 2006).

In this review, six pyridoacridine alkaloids were reported from N. carbonaria. Previous reports showed that pyridoacridine alkaloids are produced by other marine sponge including Oceanapia sp. (Eder et al., 1998), Petrosia sp (Nukoolkarn et al., 2008) and from ascidian species such as Cystodytes dellechiajei (Torres et al., 2002) and Lissoclinum cf. Badium (Clement et al., 2008). About eight motuporamines (36-42) were found in N. exigua (Williams et al., 1998; Williams et al., 2002). The occurrence of motuporamines in N. exigua only could be considered as important marker for this species. Only two phenethylguanidine derivatives were found in Neopetrosia genus. These two compounds, 7, 8dihydrotubastrine (43) and 4-deoxy-7,8dihydrotubastrine (44), were found in $N$. contignata (Petrosia cf. contignata) (Sperry and Crews, 1998). According to literatures, there are no phenethylguanidine derivatives with similar skeleton have been reported from marine origin.

More than 21 quinone and hydroquinone derivatives have been isolated from Neopetrosia genus. These derivatives were found in $N$. carbonaria, $N$. exigua, $N$. sapra, N. proxima, N. seriata and N. similis. Many quinone and hydroquinone derivatives have been obtained from Xestospongia specimen that identified to the genus level (Zhu et al., 1998; Concepción et al., 1995). Xestoquinone and halenaquinone have been found in marine sponge Adocia sp (Schmitz and Bloor, 1988).

In this study, 13 sterols compounds were found in $N$. exigua while one was obtained from $N$. contignata. Many of these sterols or others with similar skeleton have been reported from Xestospongia genus. Some of these sterols appear to be widely distributed in other organisms such as marine sponge Spirastrella inconstans (Das et al., 1993) and green alga Halimeda macroloba (Dzeha et al., 2004). The only Neopetrosia sp that has been reported to produce terpenoids is $N$. vanilla. These terpenoids (74-79) might be used as a specific marker for this species.

\section{Conclusion}

Out of 27 species of the genus Neopetrosia only Neopetrosia carbonaria, Neopetrosia contignata, Neopetrosia exigua, Neopetrosia proxima, Neopetrosia rosariensis, Neopetrosia sapra, Neopetrosia seriata, Neopetrosia similis and Neopetrosia vanilla have been studied so far. Most species of Neopetrosia haven't been investigated yet for their secondary metabolite profiles and potential bioactivities, some of taxa mentioned in the literature have been assigned to a genus level. Accordingly, it is difficult to determine such compounds as chemosystematic markers for particular species in this genus. Beside, sponge metabolites could be synthesised by the sponge itself or it is obtained from other sources such as the symbiotic microbes or the free living microbes in the marine environment (Garson et al., 1992; Lindquist et al., 2005).

Because only 9 out of 27 species of the genus Neopetrosia have been chemically studied thus far, there is significant opportunity to find out new chemical constituents from this genus.

\section{Acknowledgement}

The author is thankful to the Department of Medical Support, Al-Balqa Applied University, Al-Karak University College (AlKarak, Jordan) for providing some technical facilities to accomplish this study.

\section{References}

Abdillah S, Nurhayati APD, Nurhatika S, Setiawan E, Heffen WL. Cytotoxic and anti-oxidant activities of marine sponge diversity at Pecaron Bay Pasir Putih Situbondo East Java, Indonesia. J Pharm Res. 2013a; 6: 685-89.

Abdillah S, Wahida Ahmad R, Kamal Muzaki F, Mohd Noor N. Anti-malarial activity of Neopetrosia exigua extract in mice. J Pharm Res. 2013b; 6: 799-803.

Alvi KA, Rodriguez J, Diaz MC, Moretti R, Wilhelm RS, Lee $\mathrm{RH}$, Slate DL, et al. Protein-tyrosine kinase inhibitory properties of planar polycyclics obtained from the marine sponge Xestospongia Cf Carbonaria and from total synthesis. J Org Chem. 1993; 58: 4871-80.

Andersen RJ, Northcote PT. Xestenone, a new bicyclic C19 terpenoid from the marine sponge Xestospongia vanilla. Tetrahedron Lett. 1988; 29: 4357-60. 
Brastianos HC, Vottero E, Patrick BO, Van Soest R, Matainaho T, Mauk AG, Andersen RJ. Exiguamine A, an indoleamine2,3-dioxygenase (IDO) inhibitor isolated from the marine sponge Neopetrosia exigua. J Am Chem Soc. 2006; 128: 1604647.

Cerqueira F, Watanadilok R, Sonchaeng P, Kijjoa A, Pinto M, Van Ufford $\mathrm{HQ}$, Kroes B, et al. Clionasterol: A potent inhibitor of complement component C1. Planta Medica. 2003; 69: 174-76.

Clement JA, Kitagaki J, Yang Y, Saucedo CJ, O'Keefe BR, Weissman AM, McKee TC, et al. Discovery of new pyridoacridine alkaloids from Lissoclinum cf. badium that inhibit the ubiquitin ligase activity of $\mathrm{Hdm} 2$ and stabilize p53. Bioorg Med Chem. 2008; 16: 10022-28.

Concepción GP, Foderaro TA, Eldredge GS, Lobkovsky E, Clardy J, Barrows LR, Ireland CM. Topoisomerase IImediated DNA cleavage by adocia and xestoquinones from the Philippine sponge Xestospongia sp. J Med Chem. 1995; 38: 4503-07.

Das B, Rao SP, Srinivas K. Studies on marine chemicals, Part VI.A new clionasterol derivative from the marine sponge Spirastrella inconstans. J Nat Prod. 1993; 56: 2210-11.

De Smet P, Parys JB, Callewaert G, Weidema AF, Hill E, De Smedt $H$, Erneux $C$, et al. Xestospongin $C$ is an equally potent inhibitor of the inositol 1, 4, 5-trisphosphate receptor and the endoplasmic-reticulum $\mathrm{Ca}^{2+}$ pumps. Cell Calcium. 1999; 26: 9-13.

Dube A, Singh N, Saxena A, Lakshmi V. Anti-leishmanial potential of a marine sponge, Haliclona exigua (Kirkpatrick) against experimental visceral leishmaniasis. Parasitol Res. 2007; 101: 317-24.

Dzeha T, Jaspars M, Tabudravu J. Clionasterol, a triterpenoid from the Kenyan marine green macroalga Halimeda macroloba. Western Indian Ocean J Mar Sci. 2004; 2: 157-61.

Eder C, Schupp P, Proksch P, Wray V, Steube K, Müller CE, Frobenius W. et al. Bioactive pyridoacridine alkaloids from the micronesian sponge Oceanapia sp. J Nat Prod. 1998; 61: 301-05.

Franco LA, Macareno JL, Ocampo YC, Pájaro IP, Gaitán R. Marine sponges of the genus Neopetrosia with antiinflammatory activity. Latin American J of Pharm. 2012; 31: 976-83

Fujiwara H, Matsunaga K, Saito M, Hagiya S, Furukawa K, Nakamura H, Ohizumi Y. Halenaquinone, a novel phosphatidylinositol 3-kinase inhibitor from a marine sponge, induces apoptosis in PC12 cells. Eur J Pharmacol. 2001; 413: $37-45$.

Gafni J, Munsch JA, Lam TH, Catlin MC, Costa LG, Molinski TF, Pessah IN. Xestospongins: Potent membrane permeable blockers of the inositol 1,4,5-trisphosphate receptor. Neuron 1997; 19: 723-33.

Galeano E, Martínez A. Antimicrobial activity of marine sponges from Urabá Gulf, Colombian Caribbean region. J de Mycologie Medi. 2007; 17: 21-24.

Garson MJ, Thompson JE, Larsen RM, Battershill CN, Murphy PT, Bergquist PR. Terpenes in sponge cell membranes: Cell separation and membrane fractionation studies with the tropical marine sponge Amphimedon sp. Lipids 1992; 27: 37888.

Gorshkova IA, Gorshkov BA, Fedoreev SA, Shestak OP, Novikov VL, Stonik VA. Inhibition of membrane transport ATPases by halenaquinol, a natural cardioactive pentacyclic hydroquinone from the sponge Petrosia seriata. Comp Biochem Physiol. Part C, Pharmacol Toxicol Endocrinol. 1999; 122: 93-9.

Gorshkova IA, Gorshkov BA, Fedoreev SA, Stonik VA. Halenaquinol, a natural cardioactive pentacyclic hydroquinone, interacts with sulfhydryls on rat brain $\mathrm{Na}(+)$, K(+)-ATPase. Comp Biochem Physiol. CBP. 2001; 128: 53140 .

Hooper JNA, Kennedy JA, Soest RWM. Annotated checklist of sponges (Porifera) of the South China Sea region. The Raffles Bulletin of Zoology. 2000; Supplement: 125-207.

Iwagawa T, Nakamura K, Hirose T, Okamura H, Nakatani M. New xenicane diterpenes isolated from the acetone extract of the soft coral Xenia florida. J Nat Prod. 2000; 63: 468-72.

Kobayashi M, Hayashi K, Kawazoe K, Kitagawa I. Marine natural-products 29. Heterosigma-glycolipid-I, heterosigmaglycolipid-Ii, heterosigma-glycolipid-Iii, andheterosigmaglycolipid-Iv, 4 diacylglyceroglycolipids possessing omega-3 -polyunsaturated fatty-acid residues, from the raphidophycean dinoflagell. Chem Pharmaceut Bull. 1992; 40: 1404-10.

Kobayashi M, Kawazoe K, Kitagawa, I. Araguspongines B, C, D, E, F, G, H, and J, new vasodilative bis-1-oxaquinolizidine alkaloids from an okinawan marine sponge, Xestospongia sp. Chem Pharm Bull. 1989; 37: 1676-78.

Kobayashi M, Shimizu N, Kitagawa I, Kyogoku Y, Harada N, Uda H. Absolute stereostructures of halenaquinol and halenaquinol sulfate, pentacyclic hydroquinones from the Okinawan marine sponge Xestospongia sapra, as determined by theoretical calculation of $\mathrm{CD}$ spectra. Tetrahedron Lett. 1985; 26: 3833-36.

Kong F, Andersen RJ. Ingenamine alkaloids isolated from the sponge Xestospongia ingens: Structures and absolute configurations. Tetrahedron 1995; 51: 2895-906.

Kubota T, Kon Y, Kobayashi J. Xestosaprol C, a new pentacyclic hydroquinone sulfate from a marine sponge Xestospongia sapra. Heterocycles 2008; 76: 1571-75.

Lakshmi V, Mishra S, Srivastava S, Chaturvedi A, Srivastava M, Shukla P. Antifungal activity of marine sponge Haliclona exigua (Krikpatrick). J de Mycologie Médicale/J Med Mycol. 2010; 20: 31-35.

Laport MS, Santos OCS, Muricy G. Marine sponges: Potential sources of new antimicrobial drugs. Curr Pharm Biotechnol. 2009; 10: 86-105.

Leone PDA, Carroll AR, Towerzey L, King G, McArdle BM, Kern G, Fisher S, et al. Exiguaquinol: A novel pentacyclic hydroquinone from Neopetrosia exigua that inhibits Helicobacter pylori Murl. Org Lett. 2008; 10: 2585-88.

Li Y, Qin S, Guo YW, Gu YC, Soest RW. 9'-Epi-3beta, 3'betadimethylxestospongin $C$, a new macrocyclic diamine 
alkaloid from the Hainan sponge Neopetrosia exigua. Planta Med. 2011; 77: 179-81.

Limna Mol VP, Raveendran TV, Abhilash KR, Parameswaran PS. Inhibitory effect of Indian sponge extracts on bacterial strains and larval settlement of the barnacle, Balanus amphitrite. Int Biodeter Biodegr. 2010; 64: 506-10.

Limna Mol VP, Raveendran TV, Parameswaran PS. Antifouling activity exhibited by secondary metabolites of the marine sponge, Haliclona exigua (Kirkpatrick). Int Biodeter Biodegr. 2009; 63: 67-72.

Lindquist N, Barber PH, Weisz JB. Episymbiotic microbes as food and defence for marine isopods: Unique symbioses in a hostile environment. Proceedings. Biological Sciences / The Royal Society. 2005; 272: 1209-16.

Liu H, Mishima Y, Fujiwara T, Nagai H, Kitazawa A, Mine Y, Kobayashi $\mathrm{H}$, et al. Isolation of araguspongine $\mathrm{M}$, a new stereoisomer of an araguspongine/xestospongin alkaloid, and dopamine from the marine sponge Neopetrosia exigua collected in Palau. Mar Drugs. 2004; 2: 154-63.

Majali I, Qaralleh H, Idid S, Saad S, Susanti D, Althunibat O. Potential antimicrobial activity of marine sponge Neopetrosia exigua. J Basic Appl Res. 2015; 1: 1-13

Mayer AMS, Rodríguez AD, Taglialatela-Scafati O, Fusetani N. Marine pharmacology in 2009-2011: Marine compounds with antibacterial, antidiabetic, antifungal, anti-inflammatory, anti-protozoal, anti-tuberculosis, and antiviral activities; affecting the immune and nervous systems, and other miscellaneous mechanisms. Mar Drugs. 2013; 11: 2510.

Mora JA, Newmark F, Santos-Acevedo M, Sánchez J. Evaluation of marine sponge extracts as new sources of antimicrobial substances. Revista española de quimioterapia: Publicacion oficial de la Sociedad Española de Quimioterapia. 2008; 21: 174-79.

Mora-Cristancho JA, Arévalo-Ferro C, Ramos FA, Tello E, Duque C, Lhullier C, Falkenberg M, et al. Antifouling activities against colonizer marine bacteria of extracts from marine invertebrates collected in the Colombian Caribbean Sea and on the Brazilian coast (Santa Catarina). Zeitschrift für Naturforschung. 2011; 66: 515-26.

Morinaka BI, Molinski TF. Xestoproxamines A-C from Neopetrosia proxima: Assignment of absolute stereo structure of bis-piperidine alkaloids by integrated degradation-CD analysis. J Nat Prod. 2011; 74: 430-40.

Morris SA, Northcote PT, Andersen RJ. Triterpenoid glycosides from the Northeastern pacific marine sponge Xestospongia-vanilla. Can J Chem. 1991; 69: 1352-64.

Nakagawa M, Endo M, Tanaka N, Gen-Pei L. Structures of xestospongin $\mathrm{A}, \mathrm{B}, \mathrm{C}$ and $\mathrm{D}$, novel vasodilative compounds from marine sponge, Xestospongia exigua. Tetrahedron Lett. 1984; 25: 3227-30.

Nakamura H, Kobayashi J, Kobayashi M, Ohizumi Y, Hirata Y. Xestoquinone, a novel cardiotonic marine natural product isolated from the okinawan sea sponge Xestospongia sapra. Chem Lett. 1985; 6: 713-16.

Nakao Y, Shiroiwa T, Murayama S, Matsunaga S, Goto Y, Matsumoto $Y$, Fusetani N. Identification of renieramycin A as an anti-leishmanial substance in a marine sponge
Neopetrosia sp. Mar Drugs. 2004; 2: 55-62.

Northcote PT, Andersen RJ. Xestolide and secoxestenone, degraded triterpenoids from the sponge Xestospongia vanilla. Can J Chem. 1989; 67: 1359.

Northcote PT, Andersen RJ. Xestodiol, a new apocarotenoid from the sponge Xestospongia vanilla. J Nat Prod. 1987; 50: 1174-77.

Nukoolkarn VS, Saen-oon S, Rungrotmongkol T, Hannongbua S, Ingkaninan K, Suwanborirux K. Petrosamine, a potent anti -cholinesterase pyridoacridine alkaloid from a Thai marine sponge Petrosia n. sp. Bioorg Med Chem. 2008; 16: 6560-67.

Oku N, Matsunaga S, Soest RWM, Fusetani N. Renieramycin J, a highly cytotoxic tetrahydroisoquinoline alkaloid, from a marine sponge Neopetrosia sp. J Nat Prod. 2003; 66: 1136-39.

Orabi KY, El Sayed KA, Hamann MT, Dunbar DC, Al-Said MS, Higa T, Kelly M. Araguspongines K and L, new bioactive bis -1-oxaquinolizidine N-oxide alkaloids from Red Sea specimens of Xestospongia exigua. J Nat Prod. 2002; 65: 178285.

Proksch P, Edrada RA, Ebel R. Applied microbiology and Biotechnology. Drugs from the seas current status and microbiological implications. Vol. 59. Springer Verlag, 2002, p 125.

Qaralleh H, Idid S, Saad S, Susanti D, Mustafa B. Documentation of three sponge species belong to the family of Petrosiidae. Aust J Basic Appl Sci. 2011; 5: 1047-53.

Qaralleh H, Idid S, Saad S, Susanti D, Taher M, Khleifat K. Antifungal and antibacterial activities of four Malaysian sponge species (Petrosiidae). Journal de Mycologie Médicale / J Med Mycol. 2010; 20: 315-20.

Quirion JC, Sevenet T, Husson HP, Weniger B. Debitus C. Two new alkaloids from Xestospongia sp., a new Caledonian sponge. J Nat Prod. 1992; 55: 1505.

Ramesh P, Reddy NS, Rao TP, Venkateswarlu Y. New oxygenated africanenes from the soft coral Sinularia dissecta. J Nat Prod. 1999; 62: 1019-21.

Roll DM, Scheuer PJ, Matsumoto GK, Clardy J. Halenaquinone, A pentacyclic polyketide from a marine sponge. J Am Chem Soc. 1983; 105: 6177-78.

Roskelley CD, Williams DE, McHardy LM, Leong KG, Troussard A, Karsan A, Andersen RJ, et al. Inhibition of tumor cell invasion and angiogenesis by motuporamines. Cancer Res. 2001; 61: 6788-94.

Schmitz FJ, Bloor SJ. Xesto- and halenaquinone derivatives from a sponge, Adocia sp., from Truk lagoon. J Org Chem. 1988; 53: 3922-25.

Sorek H, Rudi A, Benayahu Y, Kashman Y. Njaoamines G and $\mathrm{H}$, two new cytotoxic polycyclic alkaloids and a tetrahydroquinolone from the marine sponge Neopetrosia sp. Tetrahedron Lett. 2007; 48: 7691-94.

Sperry S, Crews P. Dihydrotubastrines: Phenethylguanidine analogues from the indo-pacific marine sponge Petrosia cf. contignata. J Nat Prod. 1998; 61: 859-61.

Takekawa Y, Matsunaga S, Soest RWM, Fusetani N. Amphimedosides, 3-alkylpyridine glycosides from a marine 
sponge Amphimedon sp. J Nat Prod. 2006; 69: 1503-05.

Torres YR, Bugni TS, Berlinck RGS, Ireland CM, Magalhães A, Ferreira AG, Da Rocha RM. Sebastianines A and B, novel biologically active pyridoacridine alkaloids from the Brazilian ascidian cystodytes dellechiajei. J Org Chem. 2002; 67: 5429-32.

Towle KM, Chaytor JL, Liu H, Austin P, Roberge M, Roskelley CD, Vederas JC. Synthesis and biological studies of neopetrosiamides as inhibitors of cancer cell invasion. Org Biomolecular Chem. 2013; 11: 1476-81.

Tsukamoto S, Takeuchi T, Kawabata T, Kato H, Yamakuma M, Matsuo K, El-Desoky AH, et al. Halenaquinone inhibits RANKL-induced osteoclastogenesis. Bioorg Med Chem Lett. 2014; 24: 5315-17.

Venkateshwar Goud T, Srinivasa Reddy N, Raghavendra Swamy N, Siva Ram T, Venkateswarlu Y. Anti-HIV active petrosins from the marine sponge Petrosia similis. Biol Pharma Bull. 2003; 26: 1498-501.

Venkateswara Rao J, Desaiah D, Vig PJS, Venkateswarlu Y. Marine biomolecules inhibit rat brain nitric oxide synthase activity. Toxicology 1998; 129: 103-12.

Venkateswarlu Y, Farooq Biabani MA, Venkat Rami Reddy M. A new sesquiterpene from the Andaman sponge dysidea herbacia. J Nat Prod. 1994; 57: 827-28.

Wei X, Nieves K, Rodríguez AD. Neopetrosiamine A, biologically active bis-piperidine alkaloid from the Caribbean sea sponge Neopetrosia proxima. Bioorg Med Chem
Lett. 2010; 20: 5905-08.

Williams DE, Austin P, Diaz-Marrero AR, Soest RV, Matainaho $\mathrm{T}$, Roskelley CD, Roberge $\mathrm{M}$, et al. Neopetrosiamides, peptides from the marine sponge Neopetrosia sp. that inhibit amoeboid invasion by human tumor cells. Org Lett. 2005; 7: 4173-76.

Williams DE, Craig KS, Patrick B, McHardy LM, Van Soest R, Roberge M, Andersen RJ. Motuporamines, anti-invasion and anti-angiogenic alkaloids from the marine sponge Xestospongia exigua (Kirkpatrick): Isolation, structure elucidation, analogue synthesis, and conformational analysis. J Org Chem. 2002; 67: 245-58.

Williams DE, Lassota P, Andersen RJ. Motuporamines A-C, cytotoxic alkaloids isolated from the marine sponge Xestospongia exigua (kirkpatrick). J Org Chem. 1998; 63: 483841.

Winder PL, Baker HL, Linley P, Guzmán EA, Pomponi SA, Diaz MC, Reed JK, et al. Neopetrosiquinones A and B, sesquiterpene benzoquinones isolated from the deep-water sponge Neopetrosia cf. proxima. Bioorg Med Chem. 2011; 19: 6599-603.

Zhou XF, Xu T, Yang XW, Huang R, Yang B, Tang L, Liu Y. Chemical and biological aspects of marine sponges of the genus Xestospongia. Chem Biodiver. 2010; 7: 2201-27.

Zhu Y, Yoshida WY, Kelly-Borges M, et al. Noelaquinone, a new hexacyclic triazine quinone from an Indonesian Xestospongia sp. [J]. Heterocycles 1998; 49: 35560 . 


\section{Your feedback about this paper}

1. Number of times you have read this paper 0

2. Quality of paper
Excellent
Good
Ooderate
Not good

3. Your comments

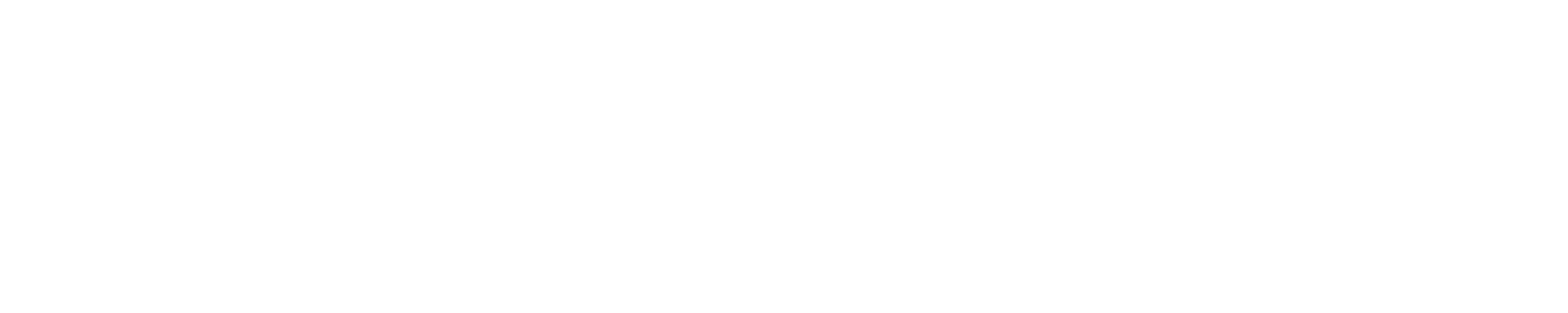

